\title{
A scalable method for identifying recombinants from unaligned sequences
}

Qian Feng $^{1}$, Kathryn Tiedje ${ }^{2,3}$, Shazia Ruybal-Pesántez ${ }^{3,4,5,6}$, Gerry Tonkin-Hill ${ }^{3,7,8}$, Michael

Duffy $^{2,3}$, Karen Day ${ }^{2,3}$, Heejung Shim ${ }^{1}$, Yao-ban Chan ${ }^{1, *}$

1 Melbourne Integrative Genomics / School of Mathematics and Statistics, The University of Melbourne, Melbourne, Australia

2 Department of Microbiology and Immunology, The University of Melbourne, Bio21 Institute,

Melbourne, Australia

3 School of BioSciences, The University of Melbourne, Bio21 Institute, Melbourne, Australia

4 Population Health and Immunity Division, Walter and Eliza Hall Institute of Medical

Research, Melbourne, Australia

5 Department of Medical Biology, The University of Melbourne, Melbourne, Australia

6 Burnet Institute, Australia

7 Bioinformatics Division, Walter and Eliza Hall Institute of Medical Research, Melbourne,

Australia

8 Parasites and Microbes, Wellcome Sanger Institute, Wellcome Genome Campus, Hinxton,

United Kingdom

*yaoban@unimelb.edu.au (YBC) 


\begin{abstract}
Recombination is a fundamental process in molecular evolution, and the identification of recombinant sequences is of major interest for biologists. However, current methods for detecting recombinants only work for aligned sequences, often require a reference panel, and do not scale well to large datasets. Thus they are not suitable for the analyses of highly diverse genes, such as the var genes of the malaria parasite Plasmodium falciparum, which are known to diversify primarily through recombination.

We introduce an algorithm to detect recombinant sequences from an unaligned dataset. Our approach can effectively handle thousands of sequences without the need of an alignment or a reference panel, offering a general tool suitable for the analysis of many different types of sequences. We demonstrate the effectiveness of our algorithm through extensive numerical simulations; in particular, it maintains its accuracy in the presence of insertions and deletions.

We apply our algorithm to a dataset of 17,335 DBL $\alpha$ types in var genes from Ghana, enabling the comparison between recombinant and non-recombinant types for the first time. We observe that sequences belonging to the same ups type or DBL $\alpha$ subclass recombine amongst themselves more frequently, and that non-recombinant DBL $\alpha$ types are more conserved than recombinant ones.
\end{abstract}

\title{
Author summary
}

Recombination is a fundamental process in molecular evolution where two genes exchange genetic material, diversifying the genes. It is important to properly model this process when reconstructing evolutionary history, and to do so we need to be able to identify recombinant genes. In this manuscript, we develop a method for this which can be applied to scenarios where current methods often fail, such as where genes are very diverse.

We specifically focus on detecting recombinants in the var genes of the malaria parasite Plasmodium falciparum. These genes influence the length and severity of malaria infection, and therefore their study is critical to the treatment and prevention of malaria. They are also highly diverse, primarily because of recombination. Our analysis of genes from a cross-sectional study in Ghana study show fundamental relations between the patterns and prevalence of recombination in these genes and other important biological categorisations. 


\section{Introduction}

Recombination, the exchange of genetic materials between two molecular sequences, is a 2 fundamental evolutionary process in viruses, prokaryotes, eukaryotes, and even between 3 kingdoms [1]. The biological mechanisms of recombination, which differ across different 4 species, lead to the creation of novel 'mosaic' sequences in which different regions have distinct 5 evolutionary histories $[2-4]$.

In human population genetics, recombination plays a central role in shaping the patterns of linkage disequilibrium, and thus recombination identification is of importance for estimating recombination rates, quantitative trait loci and association studies [5, 6]. Recombination also explains a considerable amount of the genetic diversity of human pathogens [7]-9], such as malaria [10] or protozoan parasites [11,12]. It plays a central role for parasites to escape from host immune pressures, or adapt to the effects of antiparasitic drugs. Therefore, the characterisation of recombination events is critical to the clinical treatment and prevention of 13 such diseases.

In phylogenetics, recombination breaks a central assumption, that evolution is tree-like. Not ${ }_{15}$ acknowledging recombination can result in severely misleading inferred phylogenies, e.g., the $\quad 16$ overestimation or underestimation of branch lengths [13-15]. This can be mitigated by the 17 application of phylogenetic network reconstruction methods [16]; however, these methods are 18 still in their infancy. An accurate identification of recombinant sequences would benefit these 19 methods.

Many methods have been developed for identifying recombination events and/or recombinants [3, 5, 17, 20]. They can be roughly characterised into four paradigms:

1. Distance-based methods $[1,21,-24]$ look for inversions of distance patterns among the ${ }_{23}$ sequences. They usually employ a sliding-window approach to estimate distances and are 24 generally computationally efficient.

2. Phylogenetic methods [8,25,30] look for discordant topologies in adjacent sequence segments, which is taken as a sign of recombination.

3. Compatibility methods [2] test for phylogenetic incongruence on a site-by-site basis. This type of method can be biased by many closely related sequences. 
adjacent sequence segments.

Nearly all available methods require a multiple sequence alignment, which is commonly available for population genetic datasets which have relatively low intra-population diversity. Likewise, many methods (e.g., [21, 23, 29, 30, 35]) require a reference panel of known non-recombinant sequences, which potential recombinants can be compared against. In the absence of both an alignment and a reference panel, the available methods for detecting recombinants are limited. Finally, most of the available methods do not scale well to very large datasets.

We focus on the specific application of detecting recombinants in the var genes of the malaria parasite Plasmodium falciparum. These genes express the Plasmodium falciparum erythrocyte membrane protein 1 (PfEMP1), which is the main target of the human immune response to the blood stages of infection. PfEMP1 is expressed on the surface of infected red blood cells and serves to bind host endothelial receptors [36]. It is therefore crucial for the successful proliferation and transmission [37, 38] of P. falciparum. The var genes are a large gene family (up to 60 copies per genome) [39], and high levels of diversity in the var genes have been observed in a single parasite genome, as well as small local populations [40-44]. This diversity is driven primarily by recombination [10], and so an accurate identification of var recombinants is critical to understanding the evolution of the system.

Briefly, the study of var genes has revealed a strong domain structure, including multiple Duffy-binding like domains (DBL $\alpha, \beta, \delta, \varepsilon, \gamma$, and $\mathrm{x}$ ) and cysteine-rich interdomain regions $(\mathrm{CIDR} \alpha, \beta, \gamma)[45]$. The structure of the gene itself is highly variable in both the number and the composition of these domains. Population genetic studies of var genes have focused on sequencing the DBL $\alpha$ domain, which almost always appears exactly once in a var gene. This domain has been found to be immunogenic [46] and is crucial to understanding acquired immunity and potential for vaccination [47]. Unfortunately, the DBL $\alpha$ domain is highly about their evolutionary history. the DBL $\alpha$ domain. The first systematic attempt to map out recombination in this domain was 
model (JHMM) to align a sequence to its nearest relations in a reference dataset, allowing jumps $\quad 62$ between sequences which represent recombination events. They used this method to "paint" ${ }_{63}$ each sequence according its nearest relations. This was further exploited by Tonkin-Hill et ${ }_{64}$ al. [48], who studied a large dataset of var genes around the world. They found a strong 65 geographic population structure among the genes coming from different countries.

Although these works were valuable in uncovering the recombination structure of var genes, ${ }_{67}$ there is still much work to be done. The method of Zilversmit et al. does not identify 68 recombinant sequences, only recombination events; by identifying the sequences themselves, we $\quad{ }_{69}$ can investigate the differences between the recombinants and non-recombinants, and thus 70 determine the effect of recombination on the structure and function of the gene. However, the ${ }_{71}$ diversity of the sequences and lack of an alignment and reference panel make it difficult to apply $\quad 72$ $\begin{array}{ll}\text { current methods for this task. } & 73\end{array}$

In this paper, we develop a new method to identify recombinants in a large dataset of 74 unaligned sequences. This method exploits the information produced by the JHMM method, 75 combining it with a distance-based comparison to identify recombinants. We have applied this 76 method to a large dataset of DBL $\alpha$ sequences, producing several new biological results 77 concerning the patterns of recombination in this domain. Extensive simulations also confirm the $\quad 78$ $\begin{array}{ll}\text { accuracy and applicability of our method. } & 79\end{array}$

\section{Methods}

We propose a novel method to detect recombinant sequences in a set of unaligned protein or sequences, and outputs the sequences that are identified as recombinant, their putative parents, and the corresponding breakpoints. Note that extant sequences are identified as the 'parents' of the recombinant; more accurately, we identify the descendants of the ancestral sequences which were the parents of the recombination.

Our method combines several previous methods (the JHMM method of [4] and the MAFFT algorithm of [49]) with a novel distance-based approach to identify recombinant sequences. The method consists of the following steps; see Fig 1 for a graphical overview. 


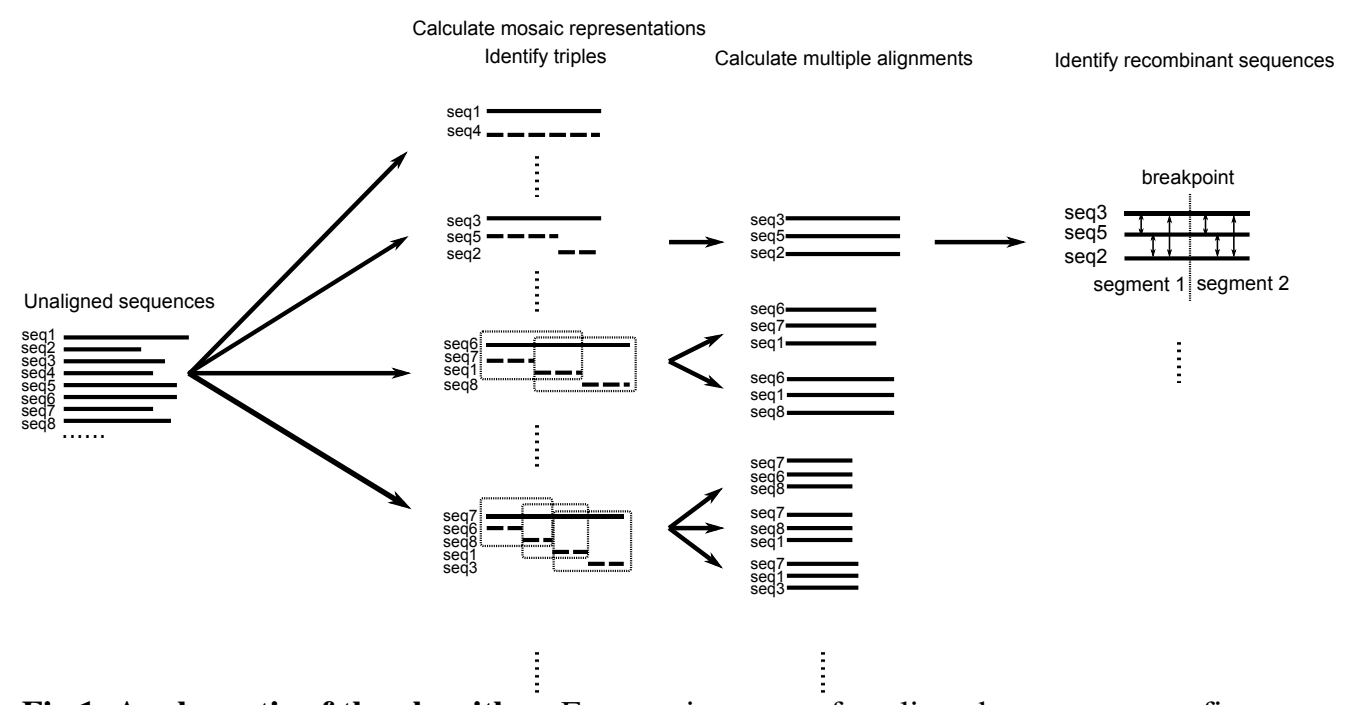

Fig 1. A schematic of the algorithm. From an input set of unaligned sequences, we first use the JHMM method to represent each sequence as a mosaic of other sequences. Next, we identify triples of segments, consisting of a recombinant segment and its two parents, and complete their alignment with the MAFFT algorithm. Finally, we identify the recombinant in each triple using a distance-based approach.

'mosaic' of segments from other sequences in the dataset.

2. From the mosaic representations, we identify triples of segments which contain a recombinant segment and its two parents. The mosaic representations provide pairwise alignments for each of these triples, which we then complete to three-way alignments with the MAFFT algorithm [49].

3. Using a distance-based approach, we identify the recombinant sequence in each triple.

We discuss each step in detail in the following sections.

\section{Calculating mosaic representations}

In this step, we use the jumping hidden Markov model of Zilversmit et al. [4] to express each $\quad 100$ sequence as a 'mosaic' combination of the other sequences in the dataset. This model was $\quad 101$ designed to uncover the patterns of recombination in a set of unaligned sequences. 102

In this model, each character in a 'target' sequence is considered to be a copy from a 103 character in a sequence in a reference set ('source' sequences). The hidden state of the Markov ${ }_{104}$ model is the (position of the) character which is copied. The copy may be imperfect, 105 representing mutation. After a character is copied, the next character in the target sequence is $\quad 106$ 
usually copied from the next character in the same source sequence. However, with small

probabilities:

- the source character may switch to any character in any position in another sequence, representing recombination;

- the model switches to an 'insertion' state, where the target character is chosen randomly ${ }_{111}$ and the source character does not move;

- the model switches to a 'deletion' state, where the source character moves forward without being copied.

If the models is in an insertion or deletion state, it continues in this state until (with a small 115 probability per character) we return to copying characters from the current source sequence. 116

We note that this model is descended from the seminal HMM of Li and Stephens [6], which ${ }_{117}$ has seen wide usage in many different applications involving recombining sequences. This 118 model is largely similar, but only works on aligned sequences, and recombination can only $\quad{ }_{119}$ switch between characters in the same position in the alignment. This restriction results in a 120 more efficient model with fewer hidden states, but one which cannot be used for unaligned $\quad{ }_{121}$ sequences.

We use the Zilversmit et al. model here by taking each sequence in our dataset in turn as the target sequence and using every other sequence in our dataset as the source sequences. We first estimate the parameters of the model, following Tonkin-Hill et al. [48]. The parameters are the probability of gap initiation $\delta$, the probability of gap extension $\varepsilon$, and the probability of recombination $\rho$. We first set $\rho$ to zero, and compute maximum likelihood estimates for $\delta$ and $\varepsilon$ with the Baum-Welch algorithm (see [50]). We then calculate the composite likelihood of all sequences for all values of $\rho$ over the interval $[0,0.1]$ under the estimated $\hat{\delta}$ and $\hat{\varepsilon}$, and choose ${ }_{129}$ the value of $\rho$ which maximises this likelihood as our estimate $\hat{\rho}$.

Finally, we calculate the Viterbi path for each target sequence to find the most probable sequence of hidden states (copied characters, insertions, and deletions). The result is a 'mosaic' ${ }_{132}$ alignment for each sequence to a series of segments from the other sequences in the dataset. An ${ }_{133}$ example of this can be seen in [4, Figure 2A]. 134

For large-scale datasets, training the JHMM model is a significant bottleneck for our method. $\quad 135$ We again follow [48], and use the Viterbi training algorithm [51] in place of the Baum-Welch to ${ }_{136}$ 
estimate $\delta$ and $\varepsilon$, and calculate the composite likelihood over 1000 randomly selected sequences $\quad{ }_{137}$ to estimate $\rho$. This allows us to analyse large datasets (such as the DBL $\alpha$ dataset in Section $\quad 138$ 'Analysis of DBL $\alpha$ sequences from a cross-sectional study in Ghana') in a practical timeframe ${ }_{139}$ with only a small loss in accuracy.

\section{Identifying recombinant triples and calculating multiple sequence}

alignments

For each sequence, the JHMM method produces an alignment of that sequence to segments from the other sequences. Whenever the source segment changes, we consider this to represent a 143 recombination event at that breakpoint. It is not necessarily the case (see below) that the target sequence in this case is the recombinant sequence, and the two source segments come from the parents of the recombination. However, we do know that the target sequence and the two source sequences form a 'recombinant triple', that is, are the two parents and the child of a recombination.

Therefore, for each breakpoint in each sequence, we identify the triple of the target sequence and the two sequences which contain the source segments before and after the breakpoint as a recombinant triple. We do this for all target sequences, resulting in a list of recombinant triples, $\quad{ }_{152}$ some of which may refer to the same recombination event. Sequences which do not infer a $\quad{ }_{153}$ breakpoint do not generate any triples.

We will apply a distance-based method to these triples to identify the true recombinant 154 sequence for each one. To calculate distances, we require a multiple alignment of the segments 155 from these three sequences. However, the JHMM method only provides a pairwise alignment of each target segment to one source segment. We take these pairwise alignments and add the corresponding segment from the remaining source sequence in the triple, using the MAFFT algorithm [49]. For each triple, this results in a multiple alignment of the segments surrounding the breakpoint. See Fig 2 for an overview of this process.

Note that we require a sufficient sequence length on either side of the breakpoint in order to calculate distances accurately. Moreover, we observe in practice that short source segments resulting from the JHMM method tend to be artifacts of the method, rather than representing multiple consecutive recombinations (see S1 Fig). To address this, we exclude triples for which the aligned segment on either side of the breakpoint is less than 10AA, which we found to be a 


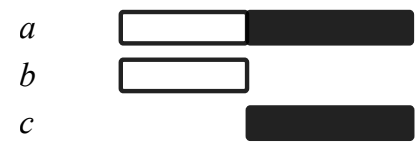

(a)

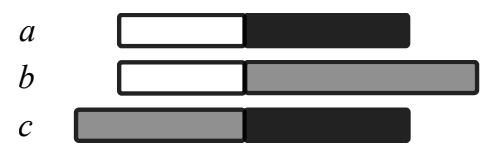

(b)

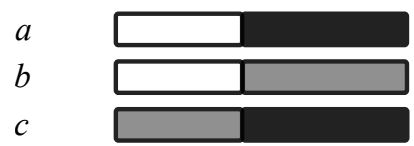

(c)

Fig 2. An example of calculating a multiple sequence alignment with MAFFT. (a): A segmental pairwise alignment generated by the JHMM method. Segments from sequence $a$ are aligned to segments from sequences $b$ and $c$ respectively. (b): Using MAFFT, we include the corresponding segment from the third sequence into the pairwise alignment on either side of the breakpoint. (c): By trimming the alignments, we generate a multiple alignment.

suitable threshold in practice.

\section{Identifying recombinant sequences}

\section{Identifiability: a phylogenetic perspective}

The main novelty in our method is the ability to identify which member of a triple is the true recombinant. It is important to note that the JHMM method does not identify the recombinant, $\quad{ }_{171}$ but instead finds the (segments of) extant sequences which are the most closely related to the target sequence.

This can be illuminated by considering an explicit phylogenetic network [16] with three aligned sequences and one recombination as an example, as shown in Fig 3 Here, we can 172 translate a phylogenetic network to the corresponding mosaic representations, assuming the JHMM method estimates the distances between sequences perfectly. It can be seen that the same mosaic structure can result from networks with different recombinants.

In fact, as discussed at length in [52], this is an unavoidable problem with the identifiability of phylogenetic networks; networks cannot be distinguished solely by the topologies of

When the phylogenetic network only consists of three sequences and one recombination (as in Fig 3], it is easy to translate the network to the JHMM output, and thus use it to find the 


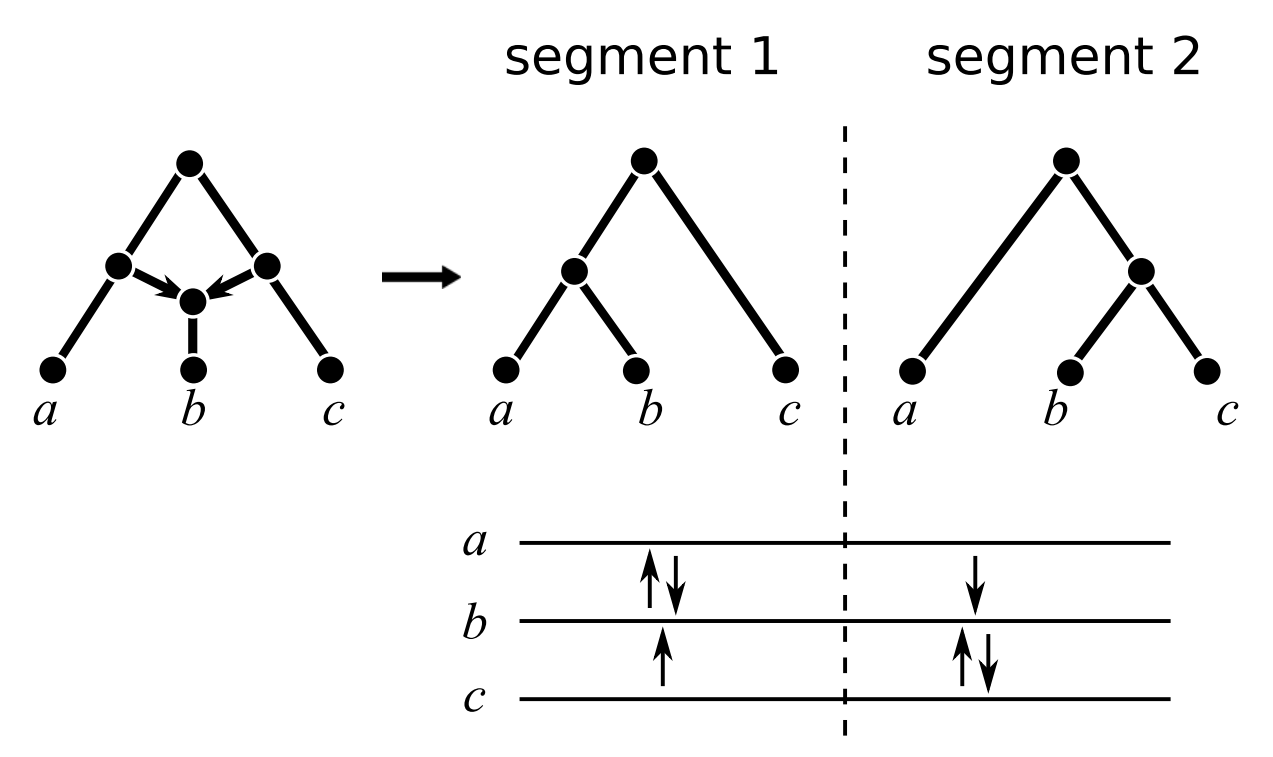

(a) sequence $b$ is the recombinant

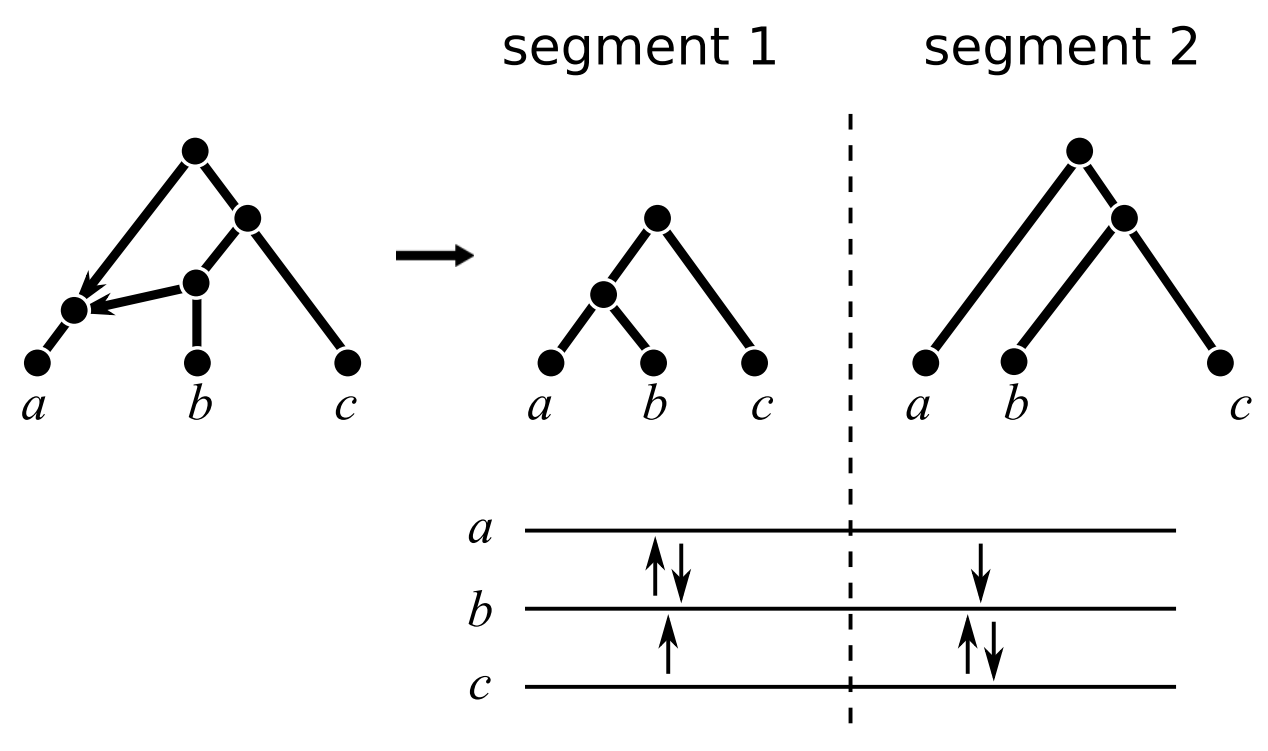

(b) sequence $a$ is the recombinant

Fig 3. Identifiability of networks from the JHMM output. Here, two networks with different recombinants produce the same profile tree topologies, and thus the same JHMM output. The JHMM output is depicted below the profile trees, with arrows from each target segment pointing to the matching source segment (so, for example, if $b$ is the target sequence, it is matched to source sequence $a$ in segment 1 and $c$ in segment 2 in both cases). Both cases produce identical JHMM output: in particular, sequence $b$ is matched to two different source sequences even though it is not necessarily the recombinant.

sequences and/or recombinations, and indeed for ancestral recombinations (predating a divergence) it's not even clear how to define an extant 'true recombinant'. To avoid this problem, ${ }_{188}$ we only identify triples of sequences as in Section "Identifying recombinant triples and 189 calculating multiple sequence alignments, , and assume that only one recombination occurs in 
the recent evolutionary history of each triple. For large datasets, we are essentially assuming that $\quad 191$ recombinations are 'sufficiently far apart' either in the network or in the genome that they do not 192 interact with each other.

From a phylogenetic perspective, we can see that when this assumption holds, identifying only triples breaks down a complicated network into repeated cases of a three sequence-one 195 recombination network, for which we can identify the recombinant. See Fig 4 for an example of 196 this.

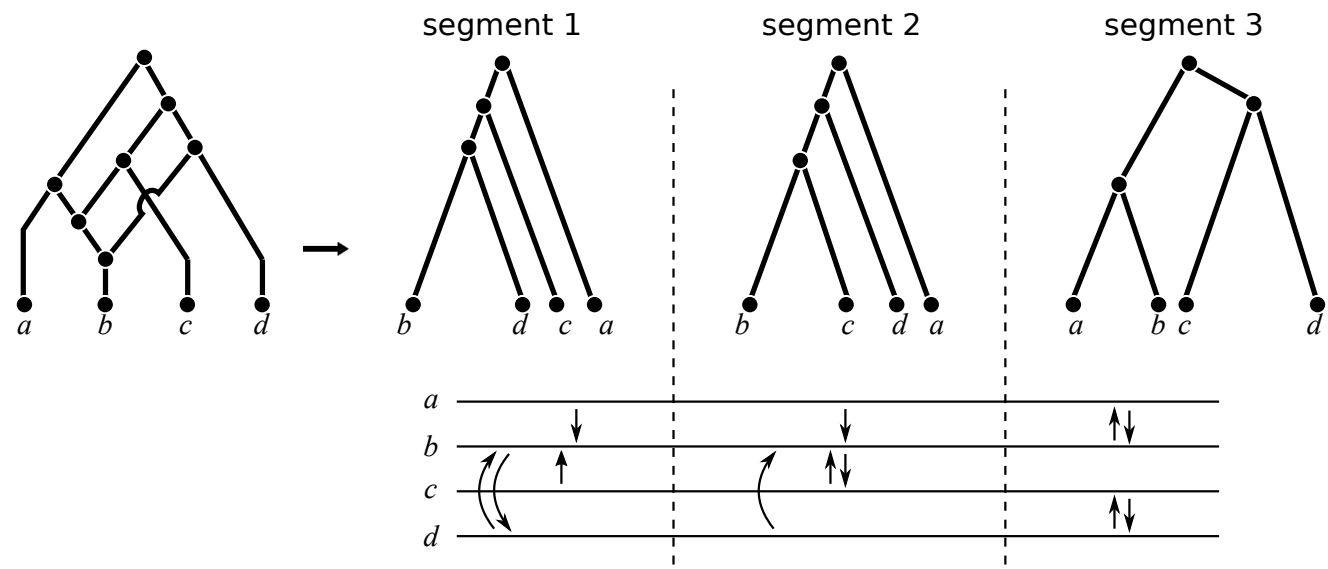

Fig 4. Decomposing a network into triples. At the first breakpoint, the triple $\{b, c, d\}$ is identified from target sequence $b$, while at the second breakpoint, $\{a, b, c\}$ is identified from sequence $b$, and $\{b, c, d\}$ from sequences $c$ and $d$. In all cases, distance-based recombinant identification will obtain the correct recombinant ( $b$ at both breakpoints).

\section{Distance-based recombinant identification}

Our algorithm is based on the well-known principle [1, 17, 32, 53] that two non-recombinant 199 sequences will have a similar evolutionary distance all along the sequence; that is, the distance between the two sequences does not change before and after a recombination breakpoint in a 201 third sequence. Conversely, the distance between a recombinant sequence and another sequence does change at a breakpoint. Using a distance-based method here allows us to avoid an 203 expensive tree or network inference step and thus scale our method to many sequences. 204

We thus calculate, for each recombinant triple $\{a, b, c\}$, the evolutionary distance between 205 each pair of segments before and after the breakpoint. We use here the BLOSUM62 distance [54,55] for amino acids and Hamming (mismatch) distance for DNA sequences (these could in principle be substituted by a large variety of ways to calculate evolutionary distance). We denote these distances by $D_{1}$ and $D_{2}$ for the first (pre-breakpoint) and second 
(post-breakpoint) segment respectively.

We then compare the distances for each pair of sequences in the triple before and after the ${ }_{211}$ breakpoint; the pair with the smallest absolute difference in distance are inferred to be the two ${ }_{212}$ non-recombinant sequences, while the third is inferred to be recombinant. Formally, we have ${ }_{213}$

$$
\text { recombinant }=\{a, b, c\} \backslash \underset{\{x, y\} \subset\{a, b, c\}}{\operatorname{argmin}}\left|D_{1}(x, y)-D_{2}(x, y)\right|
$$

This method identifies one recombinant from each recombinant triple; note that one ${ }^{214}$ recombination may generate one or more triples, but the identified recombinant from each of 215 these triples should be the same. We apply this to all triples identified above, generating a list of ${ }_{216}$ recombinants in the entire dataset and their putative parents. 217

\section{Calculating support values}

In addition to identifying recombinant sequences, we can also measure the uncertainty in our $\quad{ }_{219}$ identification by using bootstrapping. Bootstrapping in phylogenetics is a standard statistical 220 tool [56], widely used to assign uncertainties to branches on a phylogenetic tree. We use the ${ }^{221}$ same basic idea here.

For each multiple alignment of a triple, we resample characters in the alignment (columns) ${ }_{223}$ within each segment, with replacement. This provides us with a resampled alignment, and we ${ }_{224}$ generate 100 replicates per triple. We then run our distance-based method to identify the ${ }_{225}^{25}$ recombinant for each replicate. The proportion of replicates which infer the same recombinant ${ }_{226}^{22}$ as the original alignment is the support value of this detection. The larger the support value, the ${ }_{227}$ more certain we are of the detection.

Efficiency The complexity of the method is dominated by the first step of estimating the ${ }^{229}$ parameters via the Baum-Welch algorithm. As shown in [4], each iteration of the algorithm is ${ }_{230}$ $O\left(n^{2} l^{2}\right)$ in time and memory, where $n$ is the number of sequences and $l$ the length of each ${ }_{231}$ sequence. The number of iterations required is not constant, but is generally small (less than 10). ${ }^{232}$ 


\section{Results}

\section{Analysis of DBL $\alpha$ sequences from a cross-sectional study in Ghana}

Dataset We applied our method to detect recombinants and breakpoints in a dataset of DBL $\alpha \quad{ }^{235}$ sequences collected from individuals with microscopically confirmed P. falciparum infections $\quad 236$ (isolates) living in the Bongo District, in the Upper East region of Ghana (GenBank BioProject $\quad{ }_{237}$ Number: PRJNA396962) [57, 58]. Details on the study population, data collection procedures, $\quad 238$ and epidemiology have been published elsewhere [59 61]. This dataset consists of 35,591 239 previously published DBL $\alpha$ sequences collected from 161 isolates.

Preprocessing We follow the standard pipeline used in [43,48]. The DNA sequences were $\quad{ }^{241}$ first translated into protein sequences, and removed if the resulting sequence contained a stop $\quad 242$ codon. The protein sequences were then clustered with the Usearch software (v8.1.1861) [62] 243 with a $96 \%$ sequence similarity cutoff. The cluster centroids were then taken as a representative $\quad 244$ sequence for the clusters, which are known as DBL $\alpha$ types. This results in a dataset of 17,335 245 types, each of which may appear in several isolates.

Identifying recombinants We applied our method to this dataset to detect recombinant types. 247 We detected $14,801(85.4 \%)$ of the DBL $\alpha$ types to be recombinant. 248

The analysis was run on a high performance cluster at the University of Melbourne (72 $\quad 249$ Intel(R) Xeon(R) Gold 6254 CPU cores @ 3.10GHz, 768GB RAM). For estimating parameters, 250 we split the data into 578 subsets of 30 sequences each at every iteration of the Viterbi training ${ }^{251}$ algorithm, which were executed in parallel. This was also done for estimating Viterbi paths and ${ }^{252}$ identifying recombinants. The time and memory usage is summarised in Table 1 By far the $\quad{ }_{253}$ largest bottleneck is the computation of the mosaic representations of the sequences (both $\quad{ }^{254}$ parameter estimation and computation of the Viterbi paths); once this was completed, the $\quad{ }^{255}$ remaining steps are very efficient even for a dataset of this size.

Table 1. Time and memory consumption per subset ( 30 sequences).

\begin{tabular}{|l|l|l|l|}
\hline & Parameter estimation & Viterbi paths & Recombinant identification \\
\hline Time (minutes) & 644.8 & 294.9 & 2.7 \\
\hline Memory (GB) & 21.3 & 21.2 & 0.1 \\
\hline
\end{tabular}




\section{DBL $\alpha$ sequences from the same ups type recombine more frequently}

The upstream promoter sequences of each var gene can be classified into three main ups types, $\quad 258$ upsA, upsB, and upsC [41]. These ups types (not to be confused with DBL $\alpha$ types; instead, they $\quad 259$ are analogous to DBL $\alpha$ subclasses) are associated with disease severity and clinical $\quad 260$ significance [63], and thus it is crucial to investigate the behaviour of recombinants and $\quad 261$ recombinations within and between ups types.

Earlier studies on a much smaller dataset [64], based on sequence similarity, proposed that $\quad{ }_{263}$ var gene recombination preferentially occurs within the same ups type. Using our method, $\quad{ }_{264}$ which to our knowledge is the first systematic attempt to detect recombinants in var genes in $\quad 265$ natural parasite populations, we found considerable evidence supporting this hypothesis. Our ${ }_{266}$ results are summarised in Table2.

Table 2. Proportions of recombinations from the same ups types. Theoretically expected proportions, based on the base frequencies of the ups types, are given in brackets. All $p$-values are highly significant $\left(<2.2 \times 10^{-16}\right)$.

\begin{tabular}{|l|l|l|l|}
\hline & Parent-child & Parents & Family \\
\hline UpsA vs. upsB/C & $99.7 \%(85.0 \%)$ & $98.9 \%(85.0 \%)$ & $98.5 \%(77.6 \%)$ \\
\hline UpsA, B and C & $85.3 \%(50.9 \%)$ & $65.5 \%(50.9 \%)$ & $51.1 \%(30.5 \%)$ \\
\hline
\end{tabular}

Following the method of [41], we classified each DBL $\alpha$ type into one of 32 subclasses. The subclasses were then classified into either ups $\mathrm{A}$ or upsB/C types (the latter two being difficult to distinguish based on subclasses alone). For greater precision, we also developed a method to distinguish between all three types: we used BLASTP [65] to match each sequence to the closest reference sequence in [41], and then classified that sequence to the ups type of the closest reference sequence.

Having identified recombinant sequences and their putative parents, we then calculated the proportion of recombination triplets which have one parent and the child, both parents, and both parents and the child belonging to the same ups type ('Parent-child', 'Parents', and 'Family' in Table 2). In all cases, we found that the parents and/or the child of a recombination were significantly more likely ( $p<2.2 \times 10^{-16}$ from $\chi^{2}$ tests) to belong to the same ups type. This effect was most strongly noticeable when we divided the sequences only into ups $\mathrm{A}$ and B/C types; for example, the two parents and the child were in the same type $98.5 \%$ of the time, compared to a theoretical expectation of $77.6 \%$. Similar conclusions were reached when we divided the sequences into three types. Our results strongly reinforce the conclusions of earlier studies, and provide more precision with the division into three ups types. 
We also considered the proportions of identified recombinants in each ups type. We found that there was a significant difference in the proportions of recombinants in the three types $\left(p=2.193 \times 10^{-7}\right.$ from a $\chi^{2}$ test $)$, with upsA having the least proportion of recombinants, and upsC the most $(82.3 \%, 84.9 \%$, and $87.6 \%$ from $\mathrm{A}, \mathrm{B}$, and $\mathrm{C}$ respectively).

\section{Proportions of recombination differ among DBL $\alpha$ subclasses}

DBL $\alpha$ sequences can be classified according to sequence similarity into 33 subclasses

(DBL $\alpha 0.1-24, \mathrm{DBL} \alpha 1.1-8, \mathrm{DBL} \alpha 2)$. These subclasses are strongly associated with ups types; 290 however, they also provide greater resolution in dividing the sequences. We thus repeated our ${ }_{291}$ earlier analyses with regards to the subclasses.

As with ups type, we found a significant (all $p<2.2 \times 10^{-16}$ ) increase in recombinations with one parent and the child (58.8\% vs. $7.9 \%$ expected), parents (31.0\% vs. $7.9 \%$ expected), and both parents and the child (20.6\% vs. $1.0 \%$ expected) from the same subclass.

We next considered the proportions of identified recombinants in each subclass (Fig 5). We identified seven subclasses (DBL $\alpha 0.1,5$ and 11 were too high, while DBL $\alpha 0.3,8,9$ and 23 were too low) which were significantly different from the average under a Bonferroni correction for multiple testing. Of particular note is the DBL $\alpha 0.1$ subclass, which has been noted to involve more recombinations than other subclasses [10]. We suggest that these subclasses should be 300 explored further to determine if there are some biological factors that may explain these results. $\quad 301$

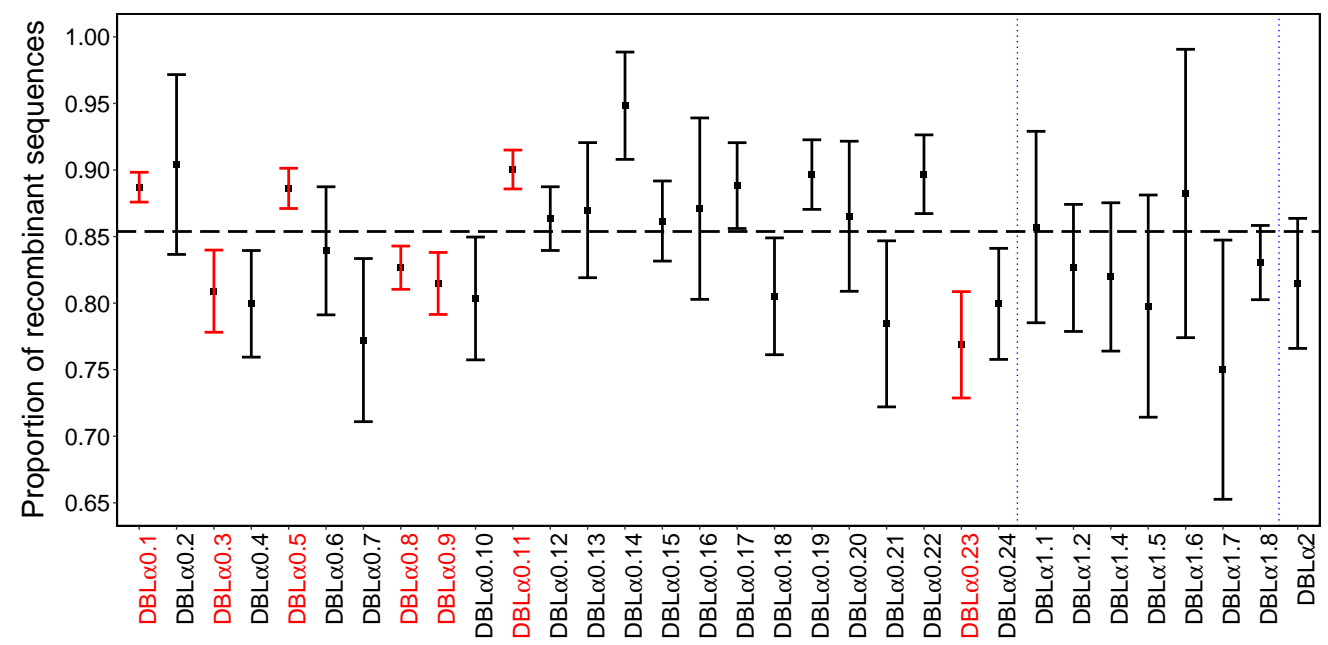

Fig 5. Proportions (and 95\% confidence intervals) of recombinants for each DBL $\alpha$ subclass. Subclasses which are significantly different from the overall average are highlighted in red. The horizontal dashed line displays the overall proportion of recombinant sequences in the entire dataset. 
We also investigated the proportion of recombinants among individual isolates, and among $\quad 302$ the two broad catchment areas in the Bongo District (Soe and Vea/Gowrie) that the isolates were ${ }_{303}$ collected from. We did not detect any significant differences here, see S1 File and S2 Fig for 304 more details.

\section{Non-recombinant DBL $\alpha$ types are more conserved than recombinant types}

It is well known [43,66] that some DBL $\alpha$ types are highly conserved, i.e., that they appear in 307 many different isolates. On the other hand, many other types only appear rarely (or even once, in 308 our large dataset). We hypothesise that non-recombinant types are more "stable" than 309 recombinants, and thus may be more highly conserved.

We investigated this hypothesis via the recombinants identified by our method. Firstly, we compared the observed frequencies of the recombinants to the non-recombinants; we found that non-recombinants occurred significantly more often in the dataset (average 4.2 vs. 3.7, $p=0.021$ from a Wilcoxon rank sum test).

We also considered if there is a difference in the proportions of frequent DBL $\alpha$ types in 315 recombinants and non-recombinants. As the frequencies of types are highly right-skewed (see $\quad 316$ S3 Fig), thus, we thresholded the frequencies at various levels to determine if there were 317 particular frequencies where an effect could be noticed. The results are in Table 3 . We found ${ }_{318}$ that for a threshold frequency of 5, there were significantly fewer frequent recombinants than $\quad 319$ non-recombinants; however, this effect becomes less noticeable for larger thresholds. This 320 suggests that there is a high proportion of recombinants which appear very few times in the ${ }_{321}$ dataset; these are potentially relatively recent recombinants, which may have not been fixed in $\quad 322$ the population.

Table 3. Proportions of frequent (larger than the threshold) recombinant and non-recombinant DBL $\alpha$ types for different thresholds.

\begin{tabular}{|l|l|l|l|l|}
\hline Threshold & $\mathbf{5}$ & $\mathbf{1 0}$ & $\mathbf{1 5}$ & $\mathbf{2 0}$ \\
\hline Recombinants & $17.5 \%$ & $4.5 \%$ & $2.1 \%$ & $1.3 \%$ \\
\hline Non-recombinants & $21.0 \%$ & $6.0 \%$ & $2.3 \%$ & $1.6 \%$ \\
\hline$P$-value $\left(\chi^{2}\right.$ test $)$ & 0.006 & 0.047 & 0.666 & 0.634 \\
\hline
\end{tabular}

\section{Breakpoint positions are associated with homology blocks}

It is known that a number of semi-conserved homology blocks (HBs) occur frequently in var $\quad 325$ genes [41]. These HBs recombine at exceedingly high rates [67,68], and are known to be useful 326 
in predicting disease severity [36]. We thus investigated the patterns of recombination in DBL $\alpha \quad 327$ types in relation to these homology blocks. 328

The positions of recombination breakpoints, as found by the JHMM method, are shown in $\quad 329$ Fig 6 Of particular note is:

- The recombination rate is not constant throughout the sequence, but displays three distinct 331 peaks spaced in roughly equal intervals. These peaks clearly correspond to the three most $\quad 332$ frequent homology blocks, HB5, 14, and 36, with the height of the peak also 333 corresponding to the frequency of the HB.

- The frequency of breakpoints drops sharply towards either end of the sequence. This is an 335 artifact of the method and does not imply that the recombination rate is lower there; we $\quad 336$ cannot recognise a recombination which is close to one end of the sequence.

This reinforces the biological theory that recombination occurs within short identical 338 segments [69]. 339

We also investigated the occurrence of HBs in the recombinant and non-recombinant 340 sequences identified by our algorithm. We discovered that the number of HBs in recombinant $\quad 341$ sequences were significantly higher than in non-recombinant sequences (5.5 vs. 5.3, 342 $p<2.2 \times 10^{-16}$ from Wilcoxon rank sum test). Furthermore, the proportion of sequences $\quad 343$ containing "important" HBs (5, 14, and 36) were also significantly different between the two 344 groups $\left(83.9 \%\right.$ vs. $78.5 \%, p=1.859 \times 10^{-11}$ from $\chi^{2}$ test), indicating that recombinants tend to 345 have more conserved building blocks. Finally, we found that recombinant sequences had higher 346 pairwise HB similarities [36] with each other than non-recombinants (0.629 vs. $0.618, \quad 347$ $p<2.2 \times 10^{-16}$ from Wilcoxon rank sum test). For more details, see S2 File 348

\section{Simulations}

\section{Simulation design}

We conducted extensive simulations to evaluate the effectiveness of our method. Our simulation $\quad 351$ protocol is as follows: 352

1. Simulate a tree (genealogy) under the coalescent (without recombination) using 353 msprime [70]. 

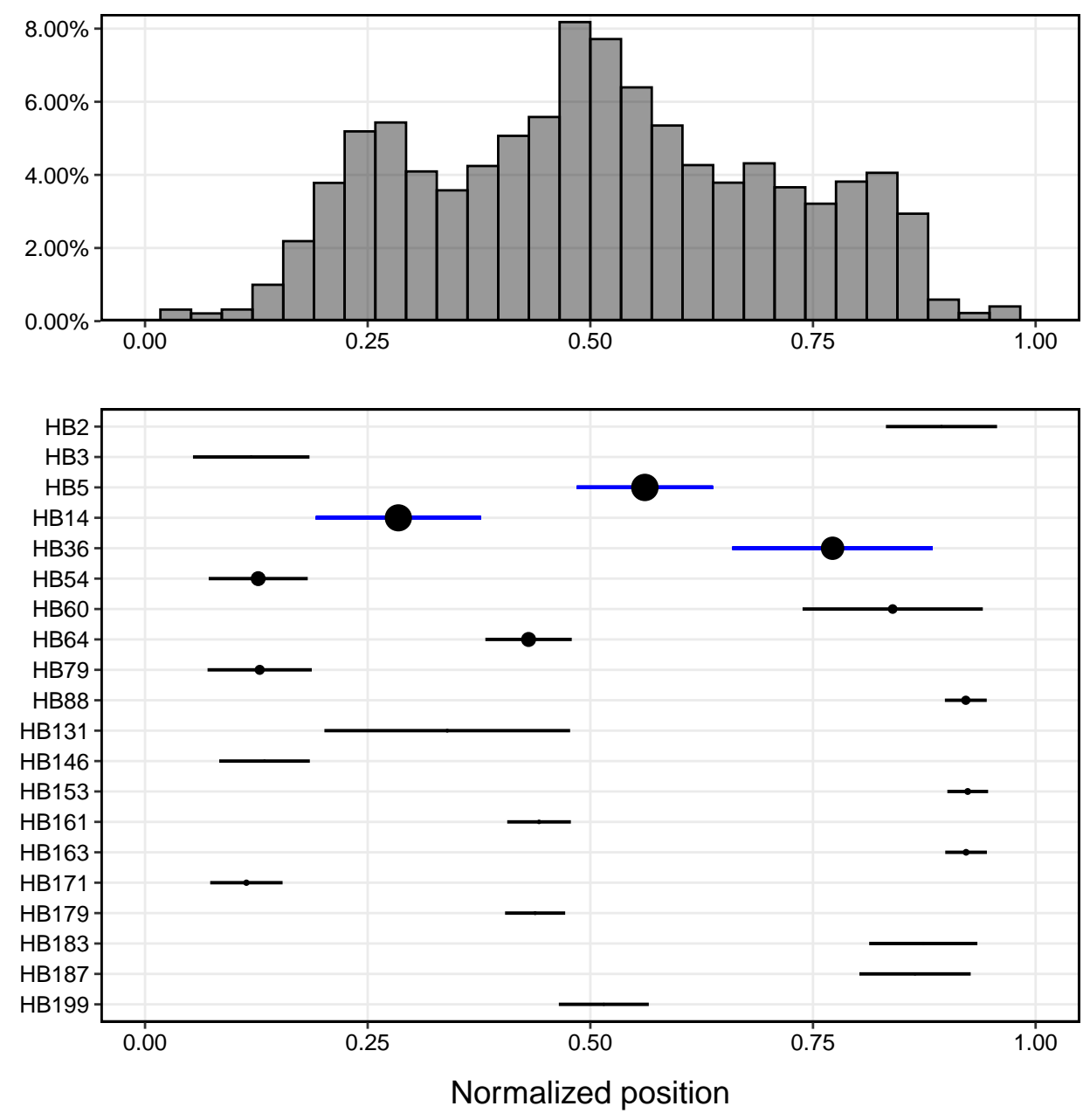

Fig 6. Positions of recombination breakpoints. (Top) The histogram of relative breakpoint positions of recombinations. (Bottom) The position of the most common homology blocks, with circle size proportional to frequency. The three most frequent homology blocks (HB5, 14, and 36) are highlighted in blue.

2. Evolve amino acid sequences from a common ancestor along the tree using Pyvolve [71]. 355

If insertions and/or deletions are required, we use INDELible [72] instead.

3. Generate recombinant sequences from two or more randomly chosen sequences in the $\quad 357$ dataset, with breakpoints chosen uniformly at random along the genome. The parent 358 sequences are removed from the dataset.

Note that we do not evolve our sequences further after the recombination step; however, since we remove the parents from the dataset, this is indistinguishable from having earlier recombinations in sequences that do not diverge. 
Table 4. General simulation parameters (no indels). We vary each parameter in turn while holding the others

fixed at the default values (in bold).

\begin{tabular}{|c|c|}
\hline Parameter & Values \\
\hline (1) Proportion of recombinant sequences (\%) & $10,20,30,40, \mathbf{5 0}, 60,70,80,90$ \\
\hline (2) Average number of recombinations per recombinant sequence & $1.0,1.1,1.2,1.3,1.4, \mathbf{1 . 5}, 1.6,1.7,1.8,1.9,2.0$ \\
\hline (3) Dataset size (sequences) & $100,150, \mathbf{2 0 0}, 250,300,350,400,450,500$ \\
\hline (4) Sequence length (AA) & $100,150, \mathbf{2 0 0}, 250,300,350,400,450,500$ \\
\hline (5) Mutation rate (substitutions/site/coalescent unit) & $0.1,0.2,0.3,0.4, \mathbf{0 . 5}, 0.6,0.7,0.8,0.9,1.0$ \\
\hline (6) Amino acid evolution model & AB [73], DAYHOFF [74], JTT [75], LG [76], MTMAM [77], WAG \\
\hline
\end{tabular}

Table 5. Indel simulation parameters (default values in bold). Insertions and deletions are simulated at the same rate, with lengths according to a negative binomial distribution with variance 10.

\begin{tabular}{|l|l|}
\hline Parameter & Values \\
\hline (7) Indel rate (expected number of indels/site/coalescent unit) & $0.1,0.2, \mathbf{0 . 3}, 0.4,0.5$ \\
\hline (8) Mean indel size (AA) & $3.7,5.2, \mathbf{6 . 0}, 6.6,7.0$ \\
\hline
\end{tabular}

There are a wide variety of parameters which could potentially affect the performance of the $\quad 365$ method. Some of these are laid out in Tables 4 and 5 . To keep our simulations tractable, we only 366 vary one parameter at a time, keeping the remainder fixed at default values. For each parameter 367 combination, we simulate 100 datasets and run our method on each dataset in turn. 368

To assess the performance of our algorithm, we calculate the sensitivity and specificity of our $\quad 369$ method for each dataset. The sensitivity is defined as the proportion of true recombinants that $\quad 370$ are correctly detected, while the specificity is the proportion of true non-recombinants that are $\quad 371$ correctly detected.

\section{Results}

Our results are shown in Figs 7f 14. Overall, it can be seen that the method enjoys good performance, with most parameter settings offering both sensitivity and specificity above $70 \%$ (and often much higher). We briefly consider the effect of each parameter in turn.

Recombinant proportion As the proportion of recombinants increases, sensitivity is stable at around $80 \%$, while specificity decreases (Fig 7). Here, more recombinant sequences result (correctly) in a higher number of recombinations detected. It appears that the proportion of true recombinants extracted from the recombinant triples remains largely the same (constant sensitivity); however, there are proportionally more false detections as the number of 
non-recombinants decreases, resulting in a lower specificity.

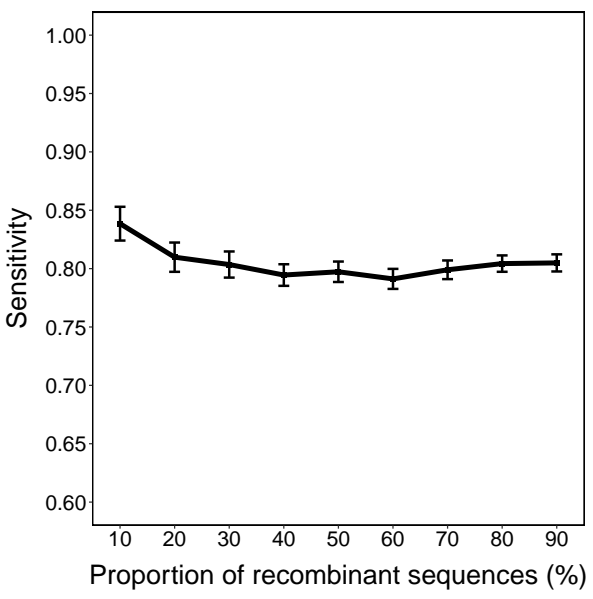

(a)

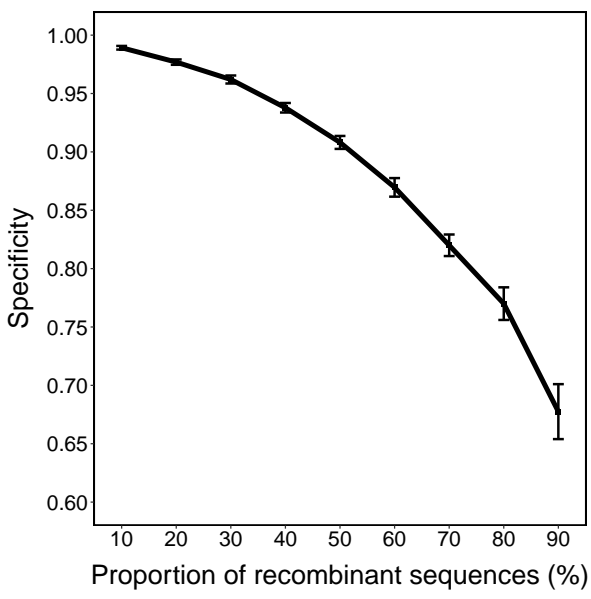

(b)

Fig 7. Mean sensitivity and specificity (with $95 \%$ confidence intervals) for varying proportions of recombinant sequences.

Number of recombinations per recombinant As shown in Fig 8 , the datasets where there $\quad 383$ are more recombinations per recombinant sequence appear to have a higher sensitivity, and $\quad 384$ slightly lower specificity. As for recombinant proportion, an increase in the number of 385 recombinations results (correctly) in more inferred recombinations; unlike that case, the number $\quad 386$ of true recombinants remains the same here. It appears that the 'extra' detections are mostly $\quad 387$ correct, which results in a greater proportion of true positives (sensitivity increases) and a $\quad 388$ relatively stable specificity.

We also conducted a further analysis by matching the distribution of the number of 390 frecombinations per recombinant to the Ghana dataset from Section "Analysis of DBL $\alpha$ sequences from a cross-sectional study in Ghanal' (see S3 File and S4 Fig for more details). Our ${ }_{392}$ results indicate that, despite a low specificity (40.0\%), a high sensitivity (89.0\%) still 393 demonstrates the applicability of our algorithm to real data.

Dataset size Dataset size does not appear to have a drastic effect on the sensitivity of the 395 method, while specificity increases slightly (see Fig 9. It is to be expected that performance 396 increases slightly as information accumulates across a larger dataset, but it is unclear why this is $\quad 397$ only expressed in the specificity here. 398 


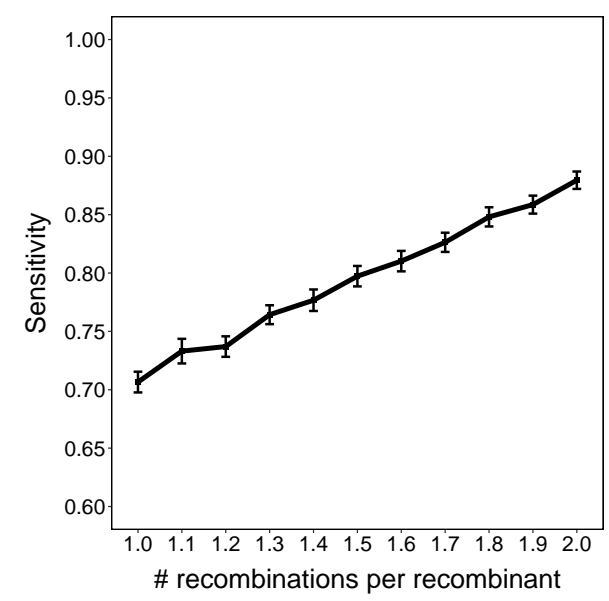

(a)

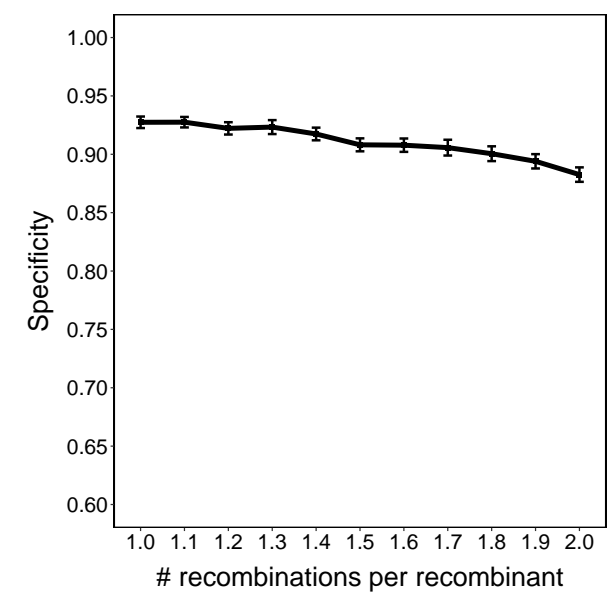

(b)

Fig 8 . Mean sensitivity and specificity (with $95 \%$ confidence intervals) for varying numbers of recombinations per recombinant sequence.

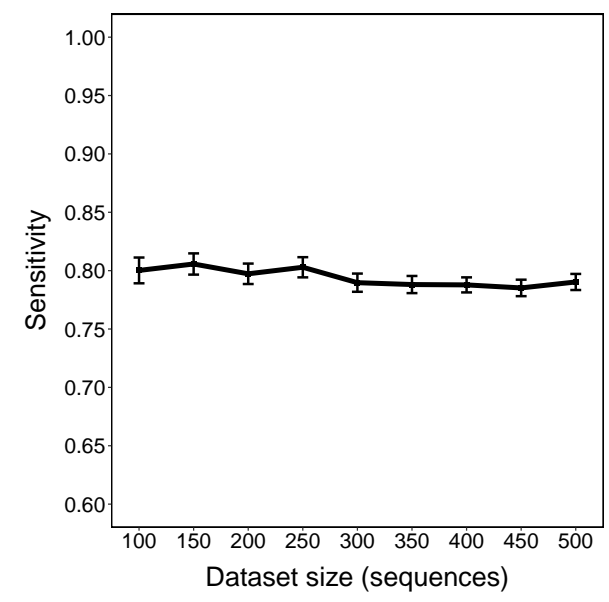

(a)

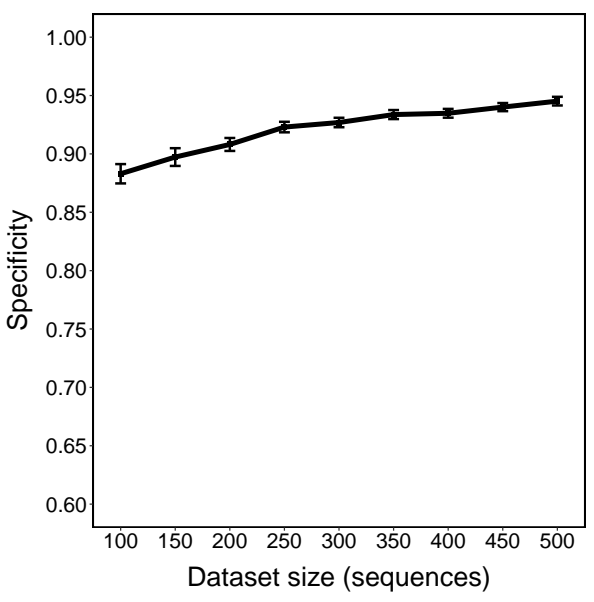

(b)

Fig 9. Mean sensitivity and specificity (with $95 \%$ confidence intervals) for varying dataset size.

Sequence length Datasets with longer sequence length have much higher sensitivity, and $\quad 399$ slightly lower specificity (Fig 10. It seems S5 Fig) that as sequence length increases, the $\quad 400$ number of recombinations detected also increases, even though the true number of 401 recombinations remains the same. This increase in detections, combined with a fixed percentage 402 of recombinants, results in a effect similar to that seen for the "number of recombinations per $\quad{ }_{403}$ recombinant": an increase in sensitivity and a slightly decreasing specificity. 


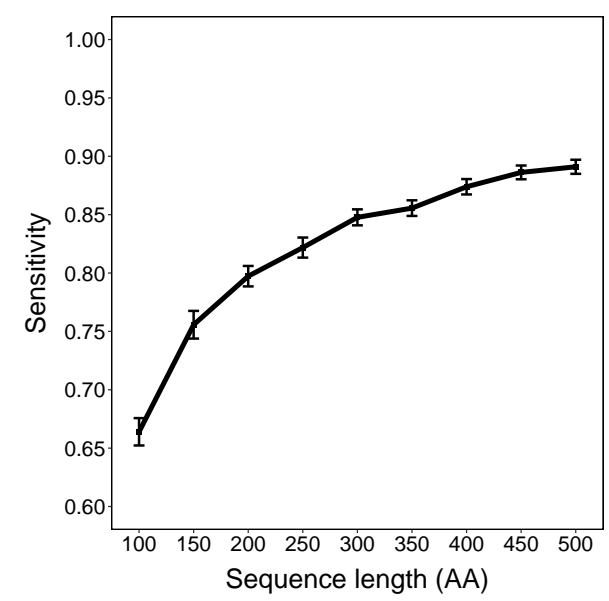

(a)

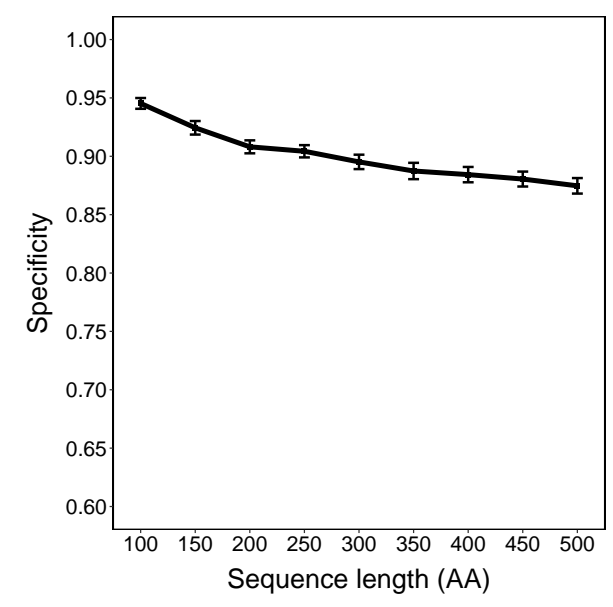

(b)

Fig 10. Mean sensitivity and specificity (with $95 \%$ confidence intervals) for varying sequence length.

Mutation rate As the mutation rate increases, the sensitivity of the method rapidly increases $\quad 405$ before levelling out (Fig 11). This makes sense, as if the number of substitutions is too low, the $\quad 406$ sequences are difficult to distinguish from each other, which makes the results from the JHMM $\quad{ }_{407}$ unreliable. Conversely, as the number of substitutions grows, it also becomes more difficult to $\quad 408$ identify sequences which are closely related to each other, resulting in a decrease in specificity. $\quad{ }_{409}$

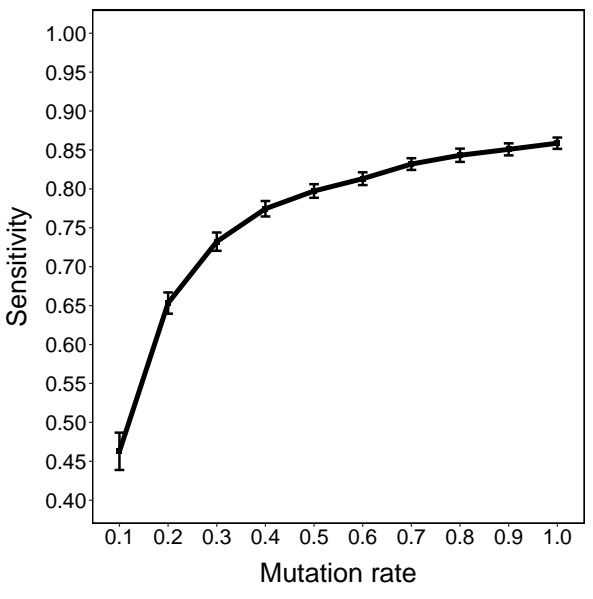

(a)

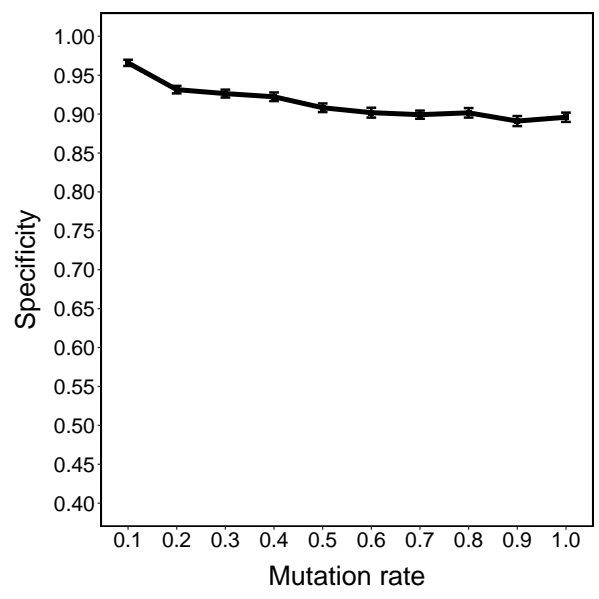

(b)

Fig 11. Mean sensitivity and specificity (with $95 \%$ confidence intervals) for varying mutation rate.

Insertion/deletion parameters An important feature of our method is its ability to accept 
(Figs 12, 13, that both sensitivity and specificity remain relatively unaffected, with a moderate ${ }_{412}$ decline in specificity as indel rate increases. This indicates that our method is robust to indels $\quad{ }_{413}$ even when the indel rate or fragment size is large. In these scenarios, existing methods which 414 only accept aligned sequences would be unable to cope.

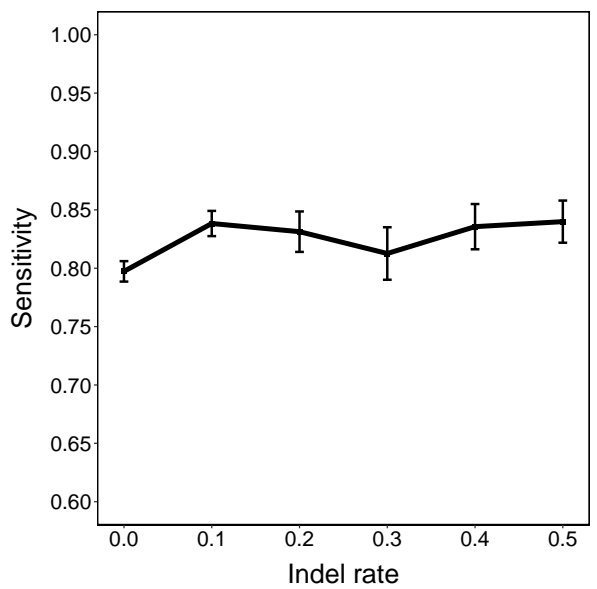

(a)

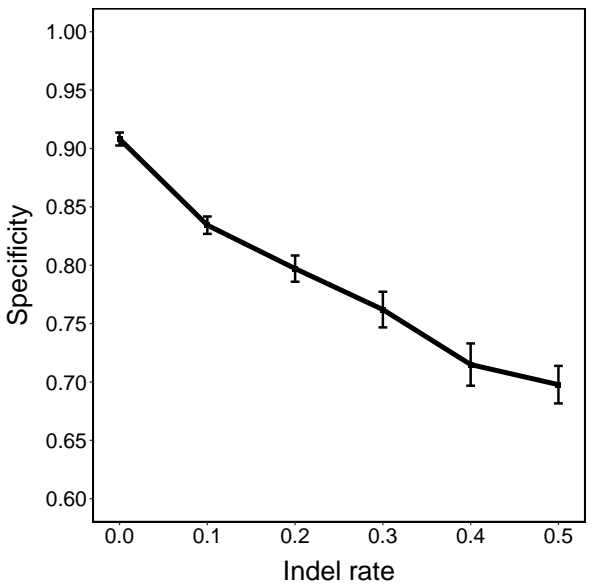

(b)

Fig 12. Mean sensitivity and specificity (with $95 \%$ confidence intervals) for varying indel rate.

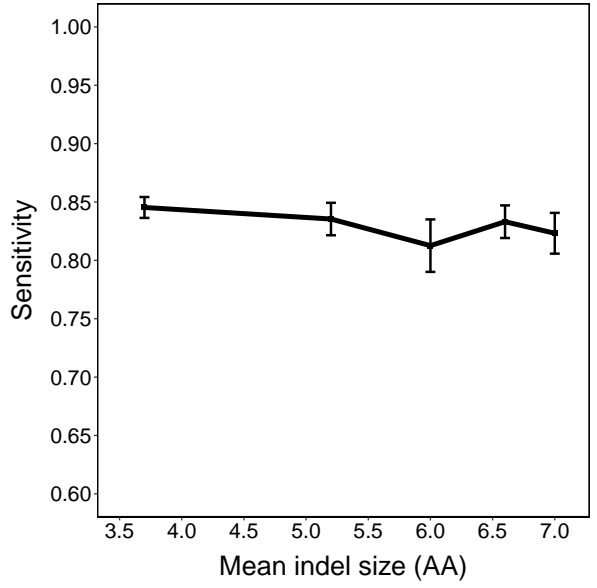

(a)

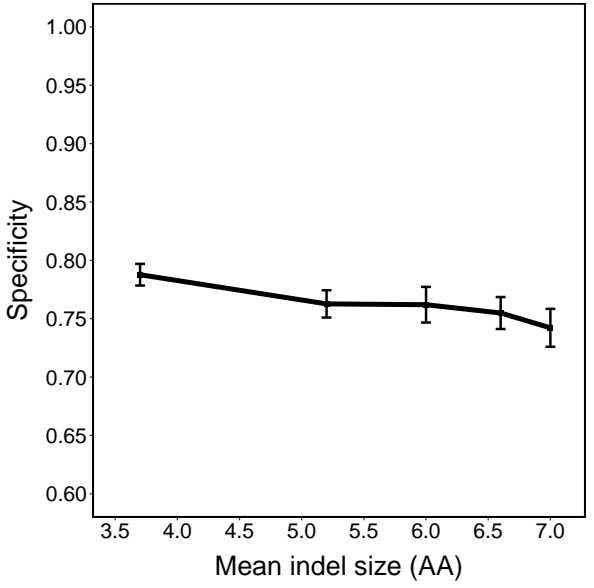

(b)

Fig 13. Mean sensitivity and specificity (with $95 \%$ confidence intervals) for varying indel size.

Other parameters The method appears to be robust to the stochastic model of amino acid ${ }_{416}$ evolution (Fig 14). 


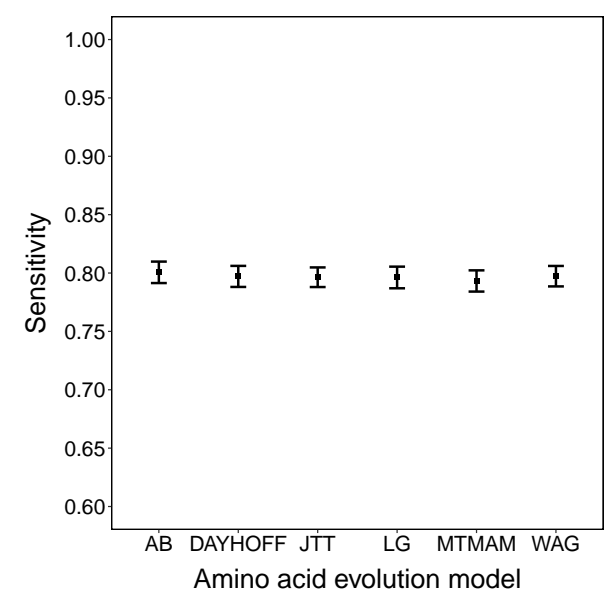

(a)

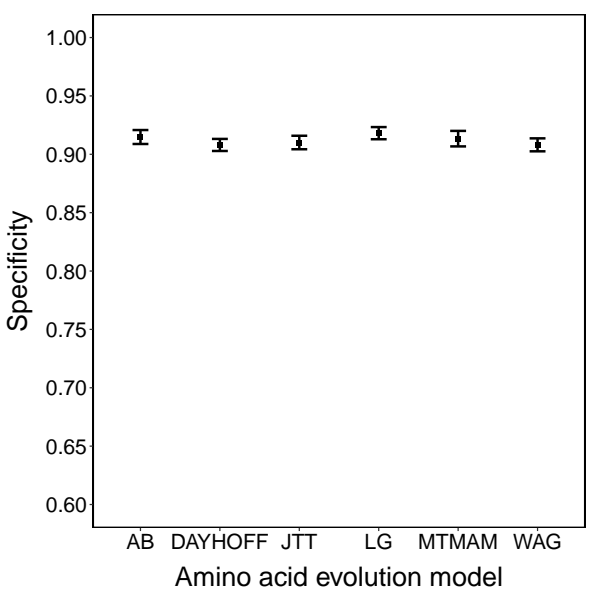

(b)

Fig 14. Mean sensitivity/specificity (with $95 \%$ confidence intervals) for each model of amino acid evolution.

\section{Support values}

In addition to detecting recombinants, we also show above how to calculate support values for $\quad{ }_{419}$ each detection using bootstrapping. Here, we verify that the calculated values are indeed 420 effective for this purpose. For our simulations, we calculate the support values for each of the ${ }_{421}$ correct detections, as well as each of the false positives. The distributions of the support values $\quad{ }_{422}$ for the default parameters are shown in Fig 15 . Here, we can see that there is a clear separation ${ }_{423}$ between the distributions of support values for the true and false positives; while the values for $\quad{ }_{424}$ both are relatively high, the support values for true detections are overall much higher. Similar $\quad{ }_{425}$ patterns are seen among all the remaining parameter settings (S6 Fig-S13 Fig). ${ }_{426}$

This suggests that we can use a threshold on the support value to refine our detections. This ${ }_{427}^{42}$ is reasonable if we wish to reduce false positives; however, in practice we found that applying a $\quad{ }_{428}$ threshold also reduced true positives (as expected) to an extent which lowered the overall $\quad{ }_{429}$ accuracy of the method, so we have elected not to apply it here. Instead, we suggest that the $\quad 430$ support value be used to assess the confidence which should be placed in individual recombinant ${ }_{431}$ detections of interest.

\section{Accuracy of the JHMM method}

The JHMM method of [4] forms a key part of our method to detect recombinants. Until now, ${ }_{434}^{4}$ there has not been a systematic study of the accuracy of this method. Two key outputs of this 435 


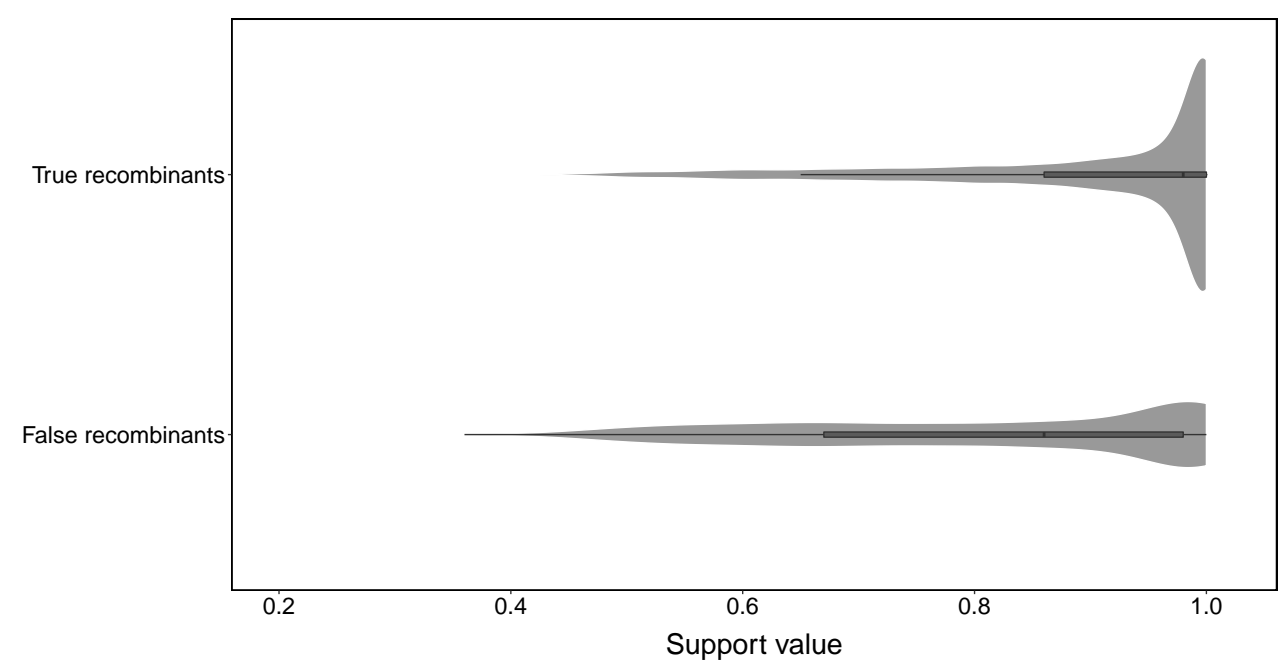

Fig 15. Distributions of support values under default parameters without indel events.

method are the locations of the inferred recombination breakpoints, and the estimated

recombination parameter $\rho$. Here, we study the accuracy of these inferences for our simulated datasets.

Recombination breakpoints For each recombination, we calculate the distance between the true and inferred breakpoints. For ease of comparison, we restrict this analysis to the case where each recombinant sequence has exactly two parents (one recombination), which avoids the problems of matching breakpoints in the same sequence to each other.

We find in general (see Fig 16 ) that the breakpoints are very accurately inferred, with $38.4 \%$ of all breakpoints inferred exactly, and $75.0 \%$ being at most $5 \mathrm{AA}$ from the true value. There is also a slight but noticeable positive bias, where the inferred breakpoints tend to be slightly larger than the true breakpoints (S14 Fig). This can be best explained by noting that the JHMM method infers the best (Viterbi) path from left to right, and recombinations are considered relatively unlikely; hence a recombination will tend to be inferred slightly later than it actually is, particularly if both parents' sequences are identical around the breakpoint.

Finally, we note that the breakpoint accuracy appears to be very robust to indel events; this is expected, since the method explicitly accounts for these events.

Recombination rate The parameter $\rho$ is directly related to the recombination rate in the dataset (although it does not provide a rate in terms of time dimension). As such, an accurate estimate of $\rho$ is valuable for molecular phylogeneticists. We observe in our simulated datasets 453 


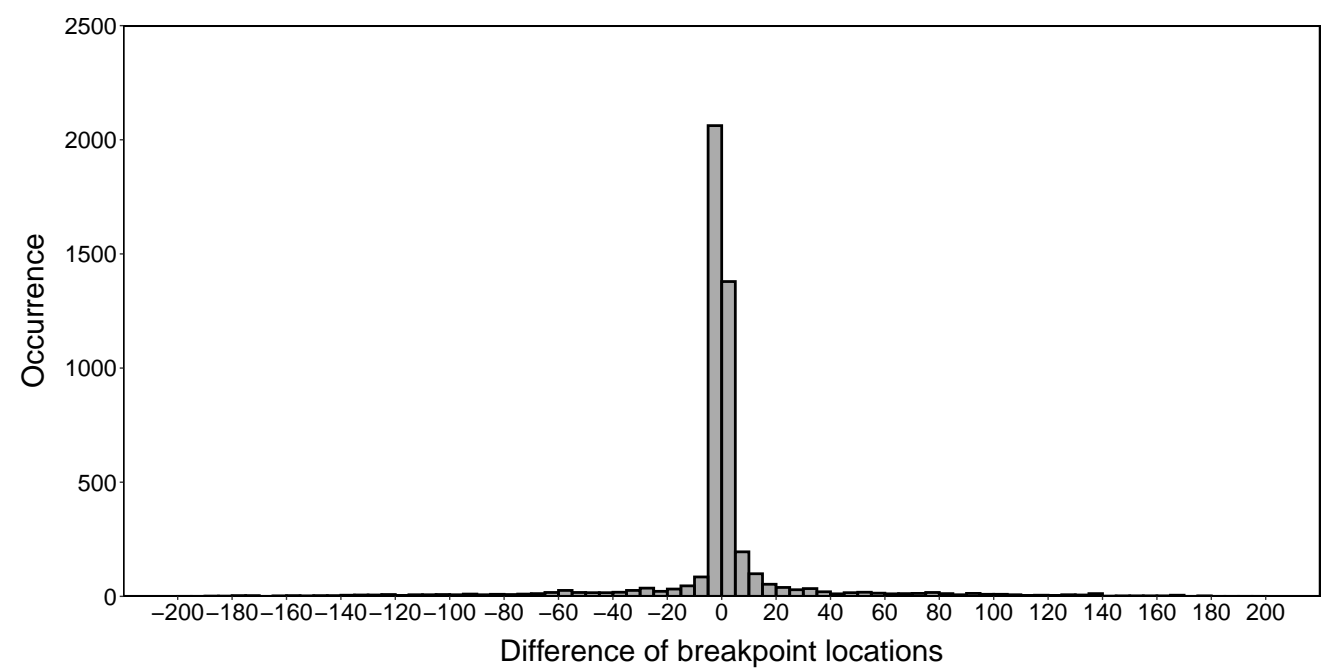

Fig 16. Breakpoint inference error of the JHMM method under default simulation parameters.

S15 Fig S18 Fig that the inferred values of $\rho$ provide an accurate estimate of the recombinaton rate.

On the other hand, the inferred $\rho$ can also be affected by mutation rate (Fig 17) and (to a lesser extent) indel events (S19 Fig S20 Fig); here, an increasing rate of non-recombination events leads to some of them being mistaken for recombination, distorting the inference of the recombination rate. This indicates that the use of the JHMM to infer the true recombination rate has the potential to be inaccurate.

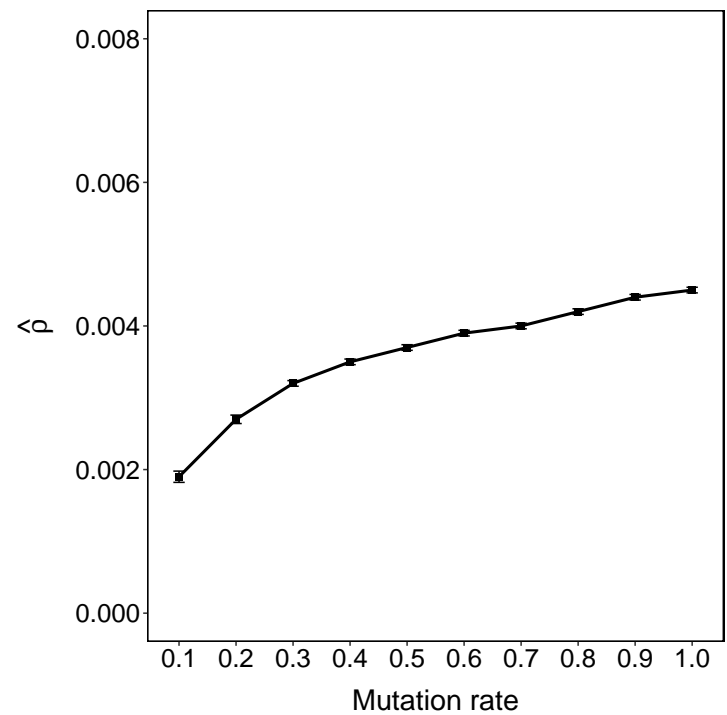

Fig 17. Estimated $\rho$ (and 95\% CI) with varying mutation rate (but constant number of recombinations). 


\section{Discussion}

In this paper, we have developed a statistical method to detect recombinant sequences from a large set of unaligned genetic sequences without a reference panel. We can also assess the reliability of the inferred recombinants with a bootstrapping-based tool. Comparisons between recombinant and non-recombinant DBL $\alpha$ types reveal a series of biologically meaningful results; for example, recombination is more frequent within ups types and DBL $\alpha$ subclasses, and non-recombinants are more conserved than recombinants. Simulations show that our method performs very well even when there is a high recombination rate, long sequences, or a large dataset. Crucially, it maintains its accuracy in the presence of insertions and deletions, 470 where methods which require an alignment would normally fail.

We note that our method is set up to detect only recent recombinants; for example, if a more ancient recombination produces a sequence that diverges into two lineages, the lineages will be preferentially matched to each other by the JHMM, and it is possible that no recombination will be detected. Note that 'recent' in this context only means that the recombinant sequence has not yet diverged; it is uncertain what timescale this corresponds to. For example, although recombination events have been reported on epidemiologically relevant timescales of several years [10], a recombinant may continue to be 'recent' for far longer than that. The Ghana dataset studied in this paper is the first of a longitudinal dataset collected over several seasons, which may give insight into the frequency and patterns of recombination on epidemiological timescales; this is the subject of current work.

Furthermore, there is an implicit assumption that recombinations do not 'interact' with each other, i.e., that they are sufficiently far apart either in the evolutionary network or in the genome that we can decompose the dataset into recombinant triples and assess those independently. This is a strong (and perhaps unrealistic, in the context of genes which have a high recombination rate) assumption which we make in order to obtain a tractable algorithm. As seen from our results, we do appear to obtain good accuracy with our detections even in cases where this assumption might not hold; assessing the exact impact of this assumption on our results is also the subject of future work.

This algorithm opens up new avenues for further analysis of var genes. In particular, the detection of (recent) recombinants and their parents will aid in the construction of phylogenetic networks. The ability to infer such a network of var genes may have important implications for 
monitoring, intervention, and diagnosis of malaria in the future.

Finally, although our methods are motivated primarily by the highly recombinant var genes, $\quad 494$ our approach is not restricted to these genes, but could be used for any genes which are 495 recombinant but lack a reliable alignment or reference panel (e.g., detecting gene fusions in the ${ }_{496}$ context of RNA sequencing in human cancer bioinformatics). The scalability of our method ${ }_{497}$ means that it will be applicable even to large datasets, thus holding great promise for broader $\quad{ }_{498}$ applications.

\section{Supporting information}

S1 File. Recombinant proportions across isolates and catchment areas. data. 
S6 Fig. Distribution of support values for varying proportions of recombinant

sequences. Red points represent the median of support values (same hereinafter).

S7 Fig. Distribution of support values for varying numbers of recombinations per

S8 Fig. Distribution of support values for varying dataset size.

S9 Fig. Distribution of support values for varying sequence length.

S10 Fig. Distribution of support values for varying mutation rate.

S11 Fig. Distribution of support values for different models of amino acid evolution.

S15 Fig. Estimated $\rho$ (and 95\% CI) for varying proportions of recombinant sequences. ${ }_{530}$ Some CIs are too short to be visible (similarly for S16 Fig $\mid$ S18 Fig $\hat{\rho}$ appears to grow linearly $\quad{ }_{531}$ with the proportion of recombinant sequences, as expected.

S16 Fig. Estimated $\rho$ (and 95\% CI) for varying number of recombinations per ${ }_{533}$ recombinant sequence. $\hat{\rho}$ appears to grow linearly with the number of recombinants per $\quad{ }_{534}$ sequence, as expected.

S17 Fig. Estimated $\rho$ (and 95\% CI) for varying dataset size. $\hat{\rho}$ decreases slightly with increasing dataset size, although the recombination rate remains constant. 
S18 Fig. Estimated $\rho$ (and 95\% CI) for varying sequence length. $\hat{\rho}$ decreases in inverse $\quad{ }_{538}$ proportion to the sequence length, as expected.

S19 Fig. Estimated $\rho$ (and 95\% CI) for varying indel rate. There is a moderate increase in 540 $\hat{\rho}$ as indel rate increases. This is unsurprising, as some of indel events are mistaken for $\quad{ }_{541}$ recombinations, distorting the inference of the recombination rate.

S20 Fig. Estimated $\rho$ (and 95\% CI) for varying indel size. Indel size (but constant indel rate) does not appear to have a drastic effect on estimated $\rho$.

\section{Acknowledgments}

We would like to thank Martine Zilversmit and Mun Hua Tan for informative discussions, and $\quad 551$

David Posada for kindly providing us the source code of the recombination detection program $\quad 552$

Chimaera.

This study was supported by the Fogarty International Center at the National Institutes of Health (Program on the Ecology and Evolution of Infectious Diseases), Grant number: 


\section{References}

1. Weiller GF. Phylogenetic profiles: a graphical method for detecting genetic recombinations in homologous sequences. Molecular Biology and Evolution. 1998;15(3):326-335.

2. Jakobsen IB, Easteal S. A program for calculating and displaying compatibility matrices as an aid in determining reticulate evolution in molecular sequences. Bioinformatics. 1996;12(4):291-295.

3. Bruen TC, Philippe H, Bryant D. A simple and robust statistical test for detecting the presence of recombination. Genetics. 2006;172(4):2665-2681.

4. Zilversmit MM, Chase EK, Chen DS, Awadalla P, Day KP, McVean G. Hypervariable antigen genes in malaria have ancient roots. BMC Evolutionary Biology. 2013;13(1):110.

5. Drysdale CM, McGraw DW, Stack CB, Stephens JC, Judson RS, Nandabalan K, et al. Complex promoter and coding region $\beta 2$-adrenergic receptor haplotypes alter receptor expression and predict in vivo responsiveness. Proceedings of the National Academy of Sciences. 2000;97(19):10483-10488.

6. Li N, Stephens M. Modeling linkage disequilibrium and identifying recombination hotspots using single-nucleotide polymorphism data. Genetics. 2003;165(4):2213-2233.

7. Robertson DL, Hahn BH, Sharp PM. Recombination in AIDS viruses. Journal of Molecular Evolution. 1995;40(3):249-259.

8. Holmes EC, Worobey M, Rambaut A. Phylogenetic evidence for recombination in dengue virus. Molecular Biology and Evolution. 1999;16(3):405-409.

9. Gibbs MJ, Armstrong JS, Gibbs AJ. Recombination in the hemagglutinin gene of the 1918 ”Spanish flu". Science. 2001;293(5536):1842-1845.

10. Claessens A, Hamilton WL, Kekre M, Otto TD, Faizullabhoy A, Rayner JC, et al. Generation of antigenic diversity in Plasmodium falciparum by structured rearrangement of Var genes during mitosis. PLoS Genetics. 2014;10(12):e1004812. 
11. Jiang H, Li N, Gopalan V, Zilversmit MM, Varma S, Nagarajan V, et al. High recombination rates and hotspots in a Plasmodium falciparum genetic cross. Genome Biology. 2011;12(4):R33.

12. Weatherly DB, Peng D, Tarleton RL. Recombination-driven generation of the largest pathogen repository of antigen variants in the protozoan Trypanosoma cruzi. BMC Genomics. 2016;17(1):729.

13. Posada D. Unveiling the molecular clock in the presence of recombination. Molecular Biology and Evolution. 2001;18(10):1976-1978.

14. Posada D, Crandall KA. The effect of recombination on the accuracy of phylogeny estimation. Journal of Molecular Evolution. 2002;54(3):396-402.

15. Kiil K, Østerlund M. CleanRecomb, a quick tool for recombination detection in SNP based cluster analysis. bioRxiv. 2018; p. 317131.

16. Huson DH, Rupp R, Scornavacca C. Phylogenetic networks: concepts, algorithms and applications. Cambridge University Press; 2010.

17. Posada D, Crandall KA. Evaluation of methods for detecting recombination from DNA sequences: computer simulations. Proceedings of the National Academy of Sciences. 2001;98(24):13757-13762.

18. Chan CX, Beiko RG, Ragan MA. A two-phase approach for detecting recombination in nucleotide sequences. arXiv preprint arXiv:07091874. 2007; .

19. Lemey P, Salemi M, Vandamme AM. The phylogenetic handbook: a practical approach to phylogenetic analysis and hypothesis testing. Cambridge University Press; 2009.

20. Haubold B, Krause L, Horn T, Pfaffelhuber P. An alignment-free test for recombination. Bioinformatics. 2013;29(24):3121-3127.

21. Siepel AC, Halpern AL, Macken C, Korber BT. A computer program designed to screen rapidly for HIV type 1 intersubtype recombinant sequences. AIDS Research and Human Retroviruses. 1995;11(11):1413-1416.

22. Huber T, Faulkner G, Hugenholtz P. Bellerophon: a program to detect chimeric sequences in multiple sequence alignments. Bioinformatics. 2004;20(14):2317-2319. 
23. Etherington GJ, Dicks J, Roberts IN. Recombination Analysis Tool (RAT): a program for the high-throughput detection of recombination. Bioinformatics. 2005;21(3):278-281.

24. Buendia P, Narasimhan G. Sliding MinPD: building evolutionary networks of serial samples via an automated recombination detection approach. Bioinformatics. 2007;23(22):2993-3000.

25. Hein J. Reconstructing evolution of sequences subject to recombination using parsimony. Mathematical Biosciences. 1990;98(2):185-200.

26. Hein J. A heuristic method to reconstruct the history of sequences subject to recombination. Journal of Molecular Evolution. 1993;36(4):396-405.

27. Grassly NC, Holmes EC. A likelihood method for the detection of selection and recombination using nucleotide sequences. Molecular Biology and Evolution. 1997;14(3):239-247.

28. Martin D, Rybicki E. RDP: detection of recombination amongst aligned sequences. Bioinformatics. 2000;16(6):562-563.

29. Martin DP, Murrell B, Golden M, Khoosal A, Muhire B. RDP4: Detection and analysis of recombination patterns in virus genomes. Virus Evolution. 2015;1(1).

30. Martin DP, Murrell B, Khoosal A, Muhire B. Detecting and analyzing genetic recombination using RDP4. Methods in Molecular Biology (Clifton, NJ). 2017;1525:433-460.

31. Sawyer S. Statistical tests for detecting gene conversion. Molecular Biology and Evolution. 1989;6(5):526-538.

32. Smith JM. Analyzing the mosaic structure of genes. Journal of Molecular Evolution. 1992;34(2):126-129.

33. Maynard Smith J, Smith NH. Detecting recombination from gene trees. Molecular Biology and Evolution. 1998;15(5):590-599.

34. Worobey M. A novel approach to detecting and measuring recombination: new insights into evolution in viruses, bacteria, and mitochondria. Molecular Biology and Evolution. 2001;18(8):1425-1434. 
35. Schultz AK, Zhang M, Bulla I, Leitner T, Korber B, Morgenstern B, et al. jpHMM: improving the reliability of recombination prediction in HIV-1. Nucleic Acids Research. 2009;37(suppl_2):W647-W651.

36. Rorick MM, Rask TS, Baskerville EB, Day KP, Pascual M. Homology blocks of Plasmodium falciparum var genes and clinically distinct forms of severe malaria in a local population. BMC Microbiology. 2013;13(1):244.

37. Scherf A, Hernandez-Rivas R, Buffet P, Bottius E, Benatar C, Pouvelle B, et al. Antigenic variation in malaria: in situ switching, relaxed and mutually exclusive transcription of var genes during intra-erythrocytic development in Plasmodium falciparum. The EMBO Journal. 1998;17(18):5418-5426.

38. Dzikowski R, Frank M, Deitsch K. Mutually exclusive expression of virulence genes by malaria parasites is regulated independently of antigen production. PLoS Pathogens. 2006;2(3).

39. Gardner MJ, Hall N, Fung E, White O, Berriman M, Hyman RW, et al. Genome sequence of the human malaria parasite Plasmodium falciparum. Nature. 2002;419(6906):498.

40. Barry AE, Leliwa-Sytek A, Tavul L, Imrie H, Migot-Nabias F, Brown SM, et al. Population genomics of the immune evasion (var) genes of Plasmodium falciparum. PLoS Pathogens. 2007;3(3):e34.

41. Rask TS, Hansen DA, Theander TG, Pedersen AG, Lavstsen T. Plasmodium falciparum erythrocyte membrane protein 1 diversity in seven genomes-divide and conquer. PLoS Computational Biology. 2010;6(9):e1000933.

42. Chen DS, Barry AE, Leliwa-Sytek A, Smith TA, Peterson I, Brown SM, et al. A molecular epidemiological study of var gene diversity to characterize the reservoir of Plasmodium falciparum in humans in Africa. PLoS One. 2011;6(2):e16629.

43. Ruybal-Pesántez S, Tiedje KE, Tonkin-Hill G, Rask TS, Kamya MR, Greenhouse B, et al. Population genomics of virulence genes of Plasmodium falciparum in clinical isolates from Uganda. Scientific Reports. 2017;7(1):11810.

44. Day KP, Artzy-Randrup Y, Tiedje KE, Rougeron V, Chen DS, Rask TS, et al. Evidence of strain structure in Plasmodium falciparum var gene repertoires in children from Gabon, 
West Africa. Proceedings of the National Academy of Sciences.

2017;114(20):E4103-E4111.

45. Bull PC, Buckee CO, Kyes S, Kortok MM, Thathy V, Guyah B, et al. Plasmodium falciparum antigenic variation. Mapping mosaic var gene sequences onto a network of shared, highly polymorphic sequence blocks. Molecular Microbiology.

2008;68(6):1519-1534.

46. Tessema SK, Nakajima R, Jasinskas A, Monk SL, Lekieffre L, Lin E, et al. Protective immunity against severe malaria in children is associated with a limited repertoire of antibodies to conserved PfEMP1 variants. Cell Host \& Microbe. 2019;26(5):579-590.

47. Sherman I. Reflections on a century of malaria biochemistry. vol. 67. Academic Press; 2011.

48. Tonkin-Hill G, Ruybal-Pesántez S, Tiedje KE, Rougeron V, Zakeri S, Pumpaibool T, et al. Global structure of the var genes encoding the major variant surface antigen of Plasmodium falciparum. PLoS Genetics. 2020. Forthcoming.

49. Katoh K, Frith MC. Adding unaligned sequences into an existing alignment using MAFFT and LAST. Bioinformatics. 2012;28(23):3144-3146.

50. Rabiner LR. A tutorial on hidden Markov models and selected applications in speech recognition. Proceedings of the IEEE. 1989;77(2):257-286.

51. Rodríguez LJ, Torres I. Comparative study of the baum-welch and viterbi training algorithms applied to read and spontaneous speech recognition. In: Iberian Conference on Pattern Recognition and Image Analysis. Springer; 2003. p. 847-857.

52. Pardi F, Scornavacca C. Reconstructible phylogenetic networks: do not distinguish the indistinguishable. PLoS Computational Biology. 2015;11(4).

53. Boni MF, Posada D, Feldman MW. An exact nonparametric method for inferring mosaic structure in sequence triplets. Genetics. 2007;176(2):1035-1047.

54. Henikoff S, Henikoff JG. Amino acid substitution matrices from protein blocks. Proceedings of the National Academy of Sciences. 1992;89(22):10915-10919. 
55. Henikoff S, Henikoff JG. Performance evaluation of amino acid substitution matrices. Proteins: Structure, Function, and Bioinformatics. 1993;17(1):49-61.

56. Felsenstein J. Confidence limits on phylogenies: an approach using the bootstrap. Evolution. 1985;39(4):783-791.

57. He Q, Pilosof S, Tiedje KE, Ruybal-Pesántez S, Artzy-Randrup Y, Baskerville EB, et al. Networks of genetic similarity reveal non-neutral processes shape strain structure in Plasmodium falciparum. Nature Communications. 2018;9(1):1-12.

58. Pilosof S, He Q, Tiedje KE, Ruybal-Pesántez S, Day KP, Pascual M. Competition for hosts modulates vast antigenic diversity to generate persistent strain structure in Plasmodium falciparum. PLoS Biology. 2019;17(6):e3000336.

59. Tiedje KE, Oduro AR, Agongo G, Anyorigiya T, Azongo D, Awine T, et al. Seasonal variation in the epidemiology of asymptomatic Plasmodium falciparum infections across two catchment areas in Bongo District, Ghana. The American Journal of Tropical Medicine and Hygiene. 2017;97(1):199-212.

60. Ruybal-Pesántez S, Tiedje KE, Rorick MM, Amenga-Etego L, Ghansah A, Oduro AR, et al. Lack of geospatial population structure yet significant linkage disequilibrium in the reservoir of Plasmodium falciparum in Bongo District, Ghana. The American Journal of Tropical Medicine and Hygiene. 2017;97(4):1180-1189.

61. Rorick MM, Artzy-Randrup Y, Ruybal-Pesántez S, Tiedje KE, Rask TS, Oduro A, et al. Signatures of competition and strain structure within the major blood-stage antigen of Plasmodium falciparum in a local community in Ghana. Ecology and evolution. 2018;8(7):3574-3588.

62. Edgar RC. Search and clustering orders of magnitude faster than BLAST. Bioinformatics. 2010;26(19):2460-2461.

63. Kaestli M, Cockburn IA, Cortés A, Baea K, Rowe JA, Beck HP. Virulence of malaria is associated with differential expression of Plasmodium falciparum var gene subgroups in a case-control study. The Journal of Infectious Diseases. 2006;193(11):1567-1574. 
64. Kraemer SM, Kyes SA, Aggarwal G, Springer AL, Nelson SO, Christodoulou Z, et al. Patterns of gene recombination shape var gene repertoires in Plasmodium falciparum: comparisons of geographically diverse isolates. BMC Genomics. 2007;8(1):45.

65. Camacho C, Coulouris G, Avagyan V, Ma N, Papadopoulos J, Bealer K, et al. BLAST+: architecture and applications. BMC Bioinformatics. 2009;10(1):421.

66. Rougeron V, Tiedje KE, Chen DS, Rask TS, Gamboa D, Maestre A, et al. Evolutionary structure of Plasmodium falciparum major variant surface antigen genes in South America: Implications for epidemic transmission and surveillance. Ecology and Evolution. 2017;7(22):9376-9390.

67. Freitas-Junior LH, Bottius E, Pirrit LA, Deitsch KW, Scheidig C, Guinet F, et al. Frequent ectopic recombination of virulence factor genes in telomeric chromosome clusters of P. falciparum. Nature. 2000;407(6807):1018-1022.

68. Taylor HM, Kyes SA, Newbold CI, et al. Var gene diversity in Plasmodium falciparum is generated by frequent recombination events. Molecular and Biochemical Parasitology. 2000;110(2):391-397.

69. Sander AF, Lavstsen T, Rask TS, Lisby M, Salanti A, Fordyce SL, et al. DNA secondary structures are associated with recombination in major Plasmodium falciparum variable surface antigen gene families. Nucleic Acids Research. 2013;42(4):2270-2281. doi:10.1093/nar/gkt1174.

70. Kelleher J, Etheridge AM, McVean G. Efficient coalescent simulation and genealogical analysis for large sample sizes. PLoS Computational Biology. 2016;12(5):e1004842.

71. Spielman SJ, Wilke CO. Pyvolve: a flexible Python module for simulating sequences along phylogenies. PLoS One. 2015;10(9):e0139047.

72. Fletcher W, Yang Z. INDELible: a flexible simulator of biological sequence evolution. Molecular Biology and Evolution. 2009;26(8):1879-1888.

73. Mirsky A, Kazandjian L, Anisimova M. Antibody-specific model of amino acid substitution for immunological inferences from alignments of antibody sequences. Molecular Biology and Evolution. 2015;32(3):806-819. 
74. Dayhoff M, Schwartz R, Orcutt B. Chapter 22: A model of evolutionary change in proteins. In: Atlas of Protein Sequence and Structure. vol. 5. National Biomedical Research Foundation Silver Spring MD; 1978. p. 345-352.

75. Jones D, Taylor W, Thornton J. A mutation data matrix for transmembrane proteins. FEBS Letters. 1994;339(3):269-275.

76. Le SQ, Gascuel O. An improved general amino acid replacement matrix. Molecular Biology and Evolution. 2008;25(7):1307-1320.

77. Yang Z, Nielsen R, Hasegawa M. Models of amino acid substitution and applications to mitochondrial protein evolution. Molecular Biology and Evolution. 1998;15(12):1600-1611.

78. Kingman JF. On the genealogy of large populations. Journal of Applied Probability. 1982;19(A):27-43. 